\title{
Article
}

\section{Mapping the circumsolar dust ring near the orbit of Venus}

Jones, M.H., Bewsher, Danielle and Brown, Daniel Stephen

Available at http://clok.uclan.ac.uk/16993/

Jones, M.H., Bewsher, Danielle ORCID: 0000-0002-6351-5170 and Brown, Daniel Stephen ORCID: 0000-0002-1618-8816 (2017) Mapping the

circumsolar dust ring near the orbit of Venus. Icarus, 288 . pp. 172-185. ISSN 0019-1035

It is advisable to refer to the publisher's version if you intend to cite from the work. http://dx.doi.org/10.1016/j.icarus.2017.01.015

For more information about UCLan's research in this area go to

http://www.uclan.ac.uk/researchgroups/ and search for < name of research Group>.

For information about Research generally at UCLan please go to http://www.uclan.ac.uk/research/

All outputs in CLoK are protected by Intellectual Property Rights law, including Copyright law. Copyright, IPR and Moral Rights for the works on this site are retained by the individual authors and/or other copyright owners. Terms and conditions for use of this material are defined in the policies page.

\section{CLoK}

Central Lancashire online Knowledge www.clok.uclan.ac.uk

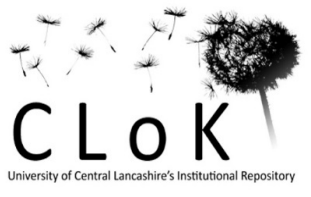




\title{
Mapping the circumsolar dust ring near the orbit of Venus
}

\author{
M.H. Jones ${ }^{1}$ \\ School of Physical Sciences, The Open University, Walton Hall, Milton Keynes, MK7 6AA, \\ United Kingdom. \\ m.h.jones@open.ac.uk \\ D. Bewsher and D.S. Brown \\ Jeremiah Horrocks Institute, University of Central Lancashire, Preston, Lancashire, \\ PR1 2HE, United Kingdom.
}

Received

accepted

${ }^{1}$ Postal address: The Open University, 351 Altrincham Road, Sharston, Manchester, M22 4UN, United Kingdom. 


\begin{abstract}
Synoptic images obtained from the HI-2 instrument on STEREO-A and -B between 2007 and 2014 have been used to further investigate the circumsolar dust ring at the orbit of Venus that was reported by Jones, Bewsher, Brown (2013). The analysis is based on high signal-to-noise ratio photometry of the zodiacal light, using data acquired over 10-day intervals, followed by a process of extracting spatial variability on scales up to about $6.5^{\circ}$. The resulting images provide information about the structure of the ring at the location where it is viewed tangentially. We identify 65 usable data sets that comprise about 11 percent of the available HI-2 data. Analysis of these images show that the orientation of the ring appears to be different to that of the orbit of Venus, with an inclination of $2.1^{\circ}$ and longitude of ascending node of $68.5^{\circ}$. We map the variation of ring density parameters in a frame of reference that is co-rotating with Venus and find a pattern suggestive of dust in a $3: 2$ orbital resonance. However, the location of the maxima of dust densities is not as expected from theoretical models, and there is some evidence that the dust density distribution in the ring has a pattern speed that differs from the mean motion of Venus.
\end{abstract}

Subject headings: Zodiacal light; Interplanetary dust; Resonances, Orbital 


\section{Introduction}

The presence of an enhancement in density of the interplanetary dust (IPD) cloud near the orbit of Venus was suggested by Leinert \& Moster (2007) based on a re-analysis of Helios data, and the existence of a circumsolar ring-like structure was confirmed by Jones, Bewsher, Brown (2013) (hereafter JBB) using optical photometry from the STEREO mission. JBB presented a subset of data acquired in 2008 and 2009 that shows a ring-like feature (with a modulation in surface brightness of about 1 percent) on angular scales of several square degrees. Importantly, this feature exhibits parallactic motion that confirms that it is associated with a physical stucture that is located close to the orbit of Venus (see Figure 2 of JBB). The other key findings presented by JBB were that; (i) density enhancements in the ring are up to about $10 \%$ of the density of the IPD cloud at the orbit of Venus (assuming the cloud density is well-modelled by the fan-model distribution of Kelsall et al. 1998), (ii) the radial density profile of the ring appears to be reasonably well modelled by two step-like density changes, and (iii) that the ring density varies with longitude in a heliocentric frame of reference that is co-rotating with the mean motion of Venus. The work described here follows on from JBB to present all usable data obtained from STEREO in the interval 2007 to 2014 to further investigate the structure of this circumsolar ring, with a particular focus on determining the orientation of the ring, and studying its azimuthal structure.

Dust grains in the interplanetary dust cloud, which are belived to originate from asteroidal and cometary sources (see e.g. Hahn et al. 2002; Nesvorný et al. 2010; Tsumura et al. 2010; Rowan-Robinson \& May 2013), are expected to have typical sizes in the range 10-100 $\mu \mathrm{m}$ (Grün et al. 1985). The orbits of such grains decay by Poynting-Robertson drag (see e.g. Burns, Lamy \& Soter 1979). In the vicinity of a planet, it is possible that gravitational perturbations can trap dust grains into exterior mean motion resonances that 
temporarily stabilise their orbits against decay, leading to a build up of dust density that manifests itself as a circumsolar ring embedded within the interplanetary dust cloud (see e.g. Weidenschilling \& Jackson 1993). This effect was first discussed in connection with the expectation that there should be a circumsolar dust ring associated with the Earth (Jackson \& Zook 1989). Analysis of infrared data from IRAS by Dermott et al. (1994) and $C O B E$ by Reach et al. (1995) led to general acceptance that such an Earth ring does exist, although a similar search for resonance features associated with Mars and Jupiter (Kuchner, Reach, \& Brown 2000) did not yield a positive detection.

Using COBE observations Kelsall et al. (1998) modelled the interplanetary dust cloud including a simple geometric model for the Earth ring. However, the ring appears faint and diffuse, and observations made from locations close to the Earth cannot easily map any azimuthal structure except from establishing that there is an increased density enhancement behind the Earth - a feature that Kelsall et al. (1998) termed the 'trailing blob'. Some further detail on the structure of the trailing cloud was presented by Reach (2010) based on in-situ measurements made using Spitzer as it drifted away from Earth on its orbit. It remains the case however, that there is considerable uncertainty about the structure of the Earth ring. The existence of a ring near the orbit of Venus that can be viewed from an exterior viewpoint presents a valuable opportunity to better understand this phenomenon.

The primary scientific purpose of the STEREO mission (Kaiser et al. 2008) is the observation of solar interaction with the heliosphere. The study presented here is based on data from the second Heliospheric Imager (HI-2) instrument, a copy of which is mounted on each STEREO spacecraft (A and B). The HI-2 is an optical imager which is sensitive to light with wavelength $(400-1000 \mathrm{~nm})$ and designed such that stray light contamination is below $\sim 10^{-14} B_{\odot}$ (where $B_{\odot}$ is the mean solar surface brightness) (Eyles et al. 2009). The scattering of sunlight by dust in the interplanetary dust (IPD) cloud gives rise to the 
zodiacal light (also termed the F-corona) and this is the dominant signal in HI-2 images. In the context of solar physics, the zodiacal light is a contaminating signal to be removed so that dynamical plasma processes in the heliosphere can be studied. Given that the purpose of HI-2 is to detect coronal mass ejections (CMEs) with a surface brightness of order one percent of the surface brightness of the zodiacal light, the instrument can also be used to search for faint features within the IPD cloud.

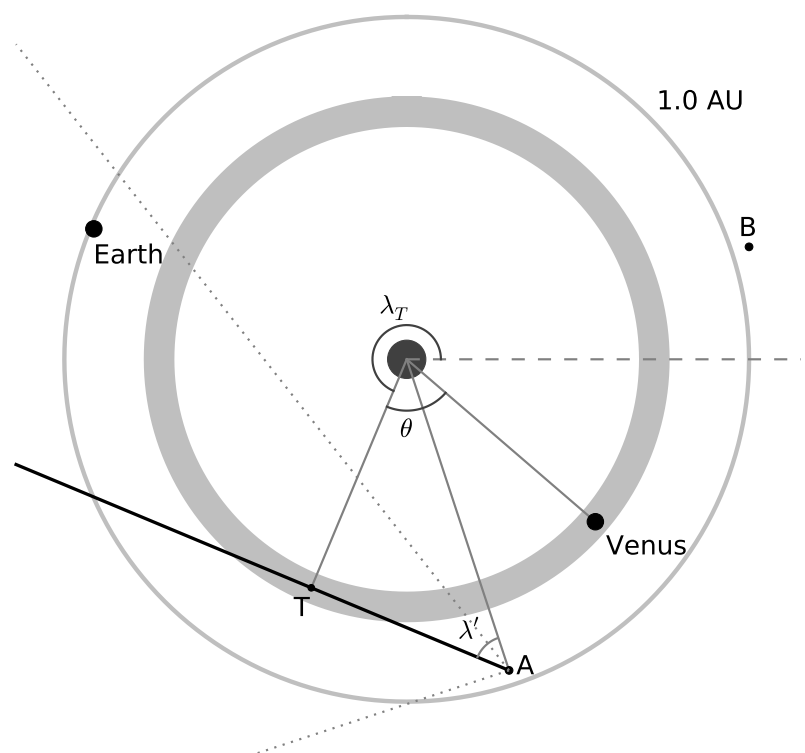

Fig. 1. - The viewing geometry of the circumsolar ring at the orbit of Venus as seen from STEREO-A and planetary positions on 2013-Feb-26 (corresponding to the mid-point of the observations that comprise data set A130221). The Sun is at the centre of the diagram, and STEREO-A and -B are labelled A and B respectively. Note that the solid line from A shows the line-of-sight to the tangent point $(\mathrm{T})$ of the Venus ring. The dashed line from the Sun shows the direction of the first point of Aries, whilst the dotted lines from A indicate the full field-of-view of the HI-2A instrument.

The typical viewing geometry that the HI-2 instruments have of the inner heliosphere is illustrated in Figure 1. Note that STEREO-A is on an orbit interior to that of the Earth, and consequently moves ahead of Earth, while the situation for STEREO-B is reversed and this spacecraft trails behind the Earth. Over time, the separation between the spacecraft and Earth increases. The data presented here correspond to the interval from launch to the 
time at which the spacecraft-Sun-Earth angle is about $180^{\circ}$ (i.e. both spacecraft are behind the Sun as viewed from Earth).

It is important to note that the key to studying a ring feature is the ability to take observations that view the tangent to the ring from an exterior viewpoint. In the case of a ring at the orbit of Venus, the tangent appears at heliocentric longitudes of $\sim 48^{\circ}$ and $\sim 42^{\circ}$ for STEREO-A and -B respectively. The field-of-view of the HI-2 instrument is $70^{\circ}$ wide, and is usually centred on locations close to the ecliptic about 53.7 East (STEREO-A) and West (STEREO-B) of the Sun. The centre of the fields-of-view of both instruments (HI-2A and HI-2B) are always within about $12^{\circ}$ of the line of sight to the tangent to the orbit of Venus, and consequently both instruments can be used to study a circumsolar ring that might be associated with Venus. Furthermore, the orbital periods of the spacecraft differ from the Keplerian period of a body near the orbit of Venus. Because of this differential rotation, when measured in a frame that is rotating with the mean motion of Venus

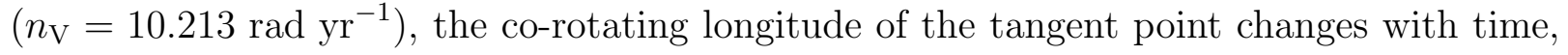
and, in principle, the azimuthal structure of the Venus ring can be investigated. (Note that hereafter the term 'co-rotating longitude' will be taken to refer to such a frame unless a different pattern speed is specified).

\section{Material and methods}

The data and methods of the study presented here follow those of JBB, but with some modifications. Here we discuss those modifications as well as providing more detailed justification for some of the choices made in JBB.

As in JBB the data used are the level-1 (L1) science images. These are the 1024 pixel-by-1024 pixel images that are obtained every 2 hours from HI-2A and HI-2B (having 
been processed on-board from exposures obtained from a CCD with dimension $2048 \times 2048$ ). Details of processing are provided in Eyles et al. (2009) and Brown, Bewsher \& Eyles (2009). Surface brightnesses in these images are reported in terms of the instrumental data number per second per CCD pixel $\left(\mathrm{DN} \mathrm{s}^{-1}\right.$ pixel $\left.^{-1}\right)$. The difference in calibration between HI-2A and HI-2B is at the level of a few percent, so we work in terms of these instrumental data values and only apply calibration corrections (as given by Tappin et al. 2015) when applying a physical model to the data.

A typical HI-2 L1 science image is shown in Figure 2. Note the presence of the zodiacal light and the stellar background in this image. Images that contain bright stellar or planetary sources result in saturation stripes running vertically across the CCD (although this is not the case for the example in Figure 2). Bright sources can also often lead to the presence of optical artefacts due to stray light reflection, which render part, or all, of a L1 science image unusable.

\subsection{Data selection}

The selection of data for inclusion in this study followed two-stages. Before any image processing was carried out, data were selected according to the criteria that: (i) the galactic plane had to be more than $30^{\circ}$ from the centre of the field-of-view, (ii) that neither the Earth nor Venus were present in the region of interest (defined below), (iii) that CME activity was low, and (iv) that there were no obvious artefacts present. Visual checks of criteria (iii) and (iv) were made by inspection of movies of the Level-2 science data ${ }^{1}$. In addition, data that met the above criteria had to be available in a set that extends for at least 10 days.

\footnotetext{
${ }^{1}$ http://www.stereo.rl.ac.uk/cgi-bin/movies.pl
} 


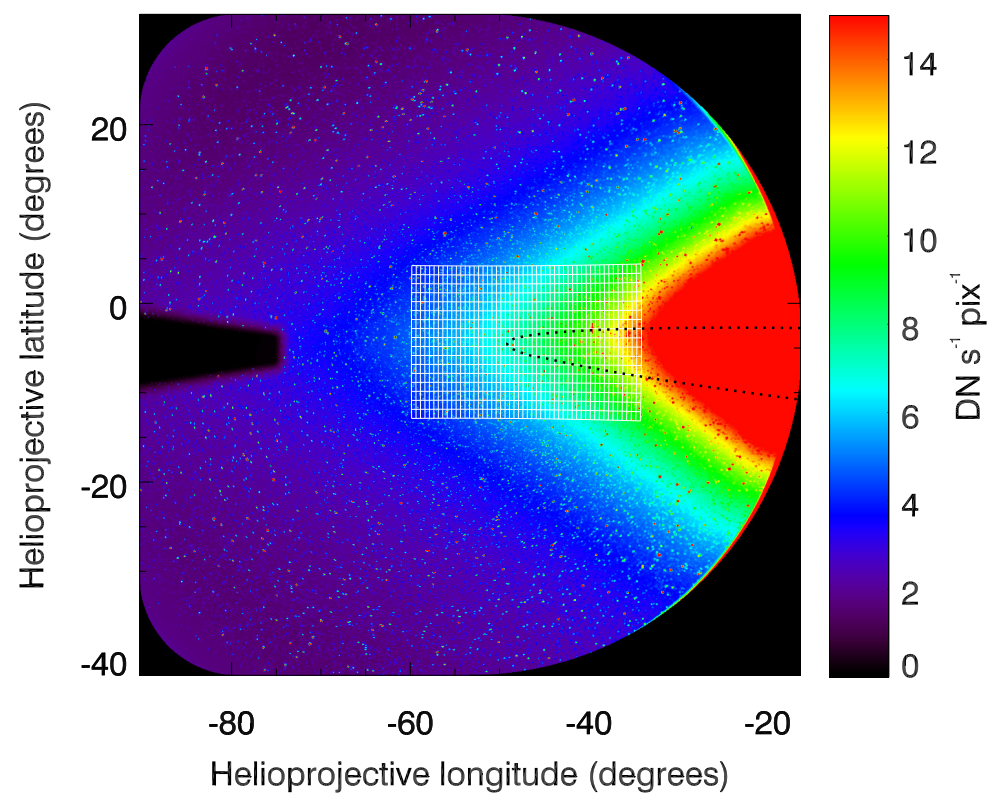

Fig. 2.- An example Level-1 science image (2008-Jun-16) from the HI-2A instrument showing the coarse grid used for the photometry used in this analysis. The field-of-view is about $70^{\circ}$ in extent. The Sun lies about $18^{\circ}$ westward of the right hand edge of the field-of-view and the orbit of Venus is indicated (black dots). The zodiacal light (i.e diffuse emission from the IPD cloud) is close to being symmetrically distributed around the plane of the ecliptic (which runs approximately horizontally across the middle of the field-of-view). The dark trapezoidal feature on the left-hand-side of the image is the Earth mask. Note that this image is labelled in helioprojective coordinates, whilst the coarse grid used for photometric measurements of the the zodiacal light is defined in terms of helioecliptic coordinates and covers a region with $35.0^{\circ} \leq \lambda^{\prime} \leq 60.0^{\circ}$ and $|\beta| \leq+8.5^{\circ}$ (see text for details). 
Using these criteria, we identified 86 data sets suitable for image processing. These are summarised in Tables 1 and 2 for STEREO-A and -B data respectively. Note that data sets are referred to by a letter denoting the spacecraft (A or B) followed by six digits that represent the year, month and date on which the observations started (such as A080616 for the STEREO-A data starting from 2008-June-16). The data sets comprise the L1 images acquired over a ten-day interval starting from 00:00 UTC on the given starting date. The second stage of data selection occured after image processing, based on the appearance of the resulting image and on a catalogue check for extended sources in the field-of-view. Data that was rejected at this stage is discussed in Section 3.1 below.

\section{2. $\quad$ Image processing}

\subsubsection{Coordinate systems}

The native coordinate system of the L1 science images is helioprojective (see e.g. Thompson 2006). It is convenient to study features related to the zodiacal cloud by reference to the ecliptic plane, and we therefore use spacecraft-centred helioecliptic

coordinates (Calabretta \& Greisen 2002): these correspond to the ecliptic coordinate system except that helioecliptic longitude $\left(\lambda^{\prime}\right)$ is measured from the position of the Sun. So, a position with ecliptic longitude $\lambda$ has $\lambda^{\prime}=\lambda-\lambda_{\odot}$ where $\lambda_{\odot}$ is the ecliptic longitude of the Sun, while helioecliptic latitude $(\beta)$ corresponds to ecliptic latitude.

\subsubsection{Photometric analysis}

In the first stage of processing, a set of L1 images are overlaid onto a coarse grid with extent $35.0^{\circ} \leq \lambda^{\prime} \leq 60.0^{\circ}$ (STEREO-A) or $-53.0^{\circ} \leq \lambda^{\prime} \leq-28.0^{\circ}$ (STEREO-B) and $|\beta| \leq 8.5^{\circ}$. The positioning of the grid for STEREO-B differs from that used in JBB to 
ensure that the location of the tangent to the orbit of Venus occurs in a similar position relative to the grid for observations made from both spacecraft. The grid has bins that are $0.5^{\circ} \times 1.0^{\circ}$ in wide in longitude and latitude respectively. The location and extent of this grid is shown for the example HI-2A image in Figure 2. Data from HI-2 images are allocated to these bins based on the helioecliptic coordinates at the centres of the L1 image pixels. These values are sorted into a sequence of increasing surface brightness, to generate a cumulative probability distribution for every bin. Data that are rejected (most commonly due to saturation stripes) are logged, so that decisions can be made on the reliability of the coarse grid data on the basis of the fraction of missing data. Each data set contains images acquired over 10 consecutive days, so, up to $120 \mathrm{HI}-2$ images are mapped onto the coarse grid, and each bin of the grid typically contains $\sim 10^{4}$ pixel values.

A key consideration is how to determine the surface brightness of zodiacal light from the measured distribution of surface brightnesses in a bin. Examples of the cumulative distribution functions of pixel values in three bins are shown (in black) in Figure 3. It is expected that the distribution of pixels should have two components: one due to zodiacal light, that affects all pixels, and another due to the stellar background that is present only in some pixels. The zodiacal component is expected to be close to a normal distribution, while the stellar component will be highly skewed. This is borne out by Figure 3, in which cumulative normal distributions, with different means but with the same value of standard deviation are overlaid (in red). There is a good match between these and the observed cumulative distribution functions for probability values between 0.05 and 0.35 . At higher probability values, the effect of the stellar component dominates. It is important to note that the variation between bins at high probability values represents different distributions of bright stars. Recognising this, we note that there is no need to explicitly mask out such stars from the L1 images prior to photometric analysis. 
To estimate the contribution from zodiacal light we adopt a probability value $P_{z}$ that provides a measure of the mean of the normally distributed component. It is important to note that the aim of the photometric analysis is to determine the spatial variation in surface brightness rather than absolute values, and it is only necessary that $P_{z}$ should provide a good estimate of the surface brightness to within a constant offset value. For simplicity, we have adopted $P_{z}=0.25$ (i.e. the first quartile, which is offset from the mean by 0.6745 times the standard deviation). Further investigation that the choice of $P_{z}$ has on the residual stellar background in processed images is considered further in Section 2.2.5

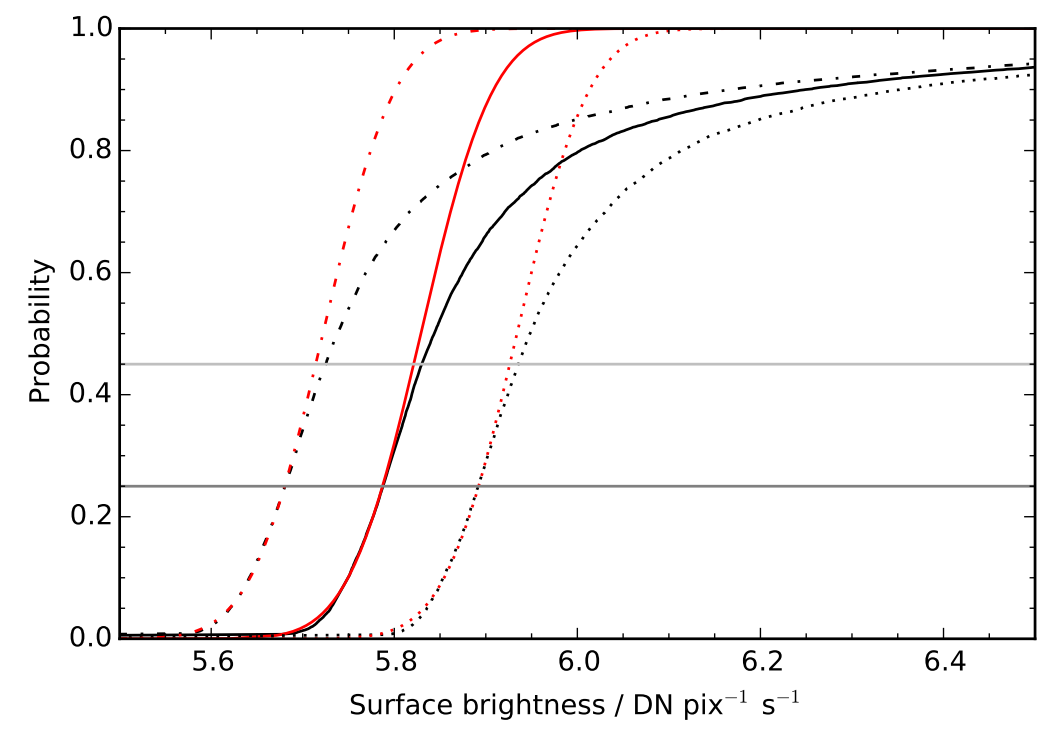

Fig. 3.- The cumulative probability distributions of pixel values in bins with centres at $\left(\lambda^{\prime}, \beta\right)$ of $(53.75,0.0)$ (dotted black line), $(54.25,0.0)$ (solid black line) and $(54.75,0.0)$ (dashdot line). The numbers of pixels in these bins are 10974, 10940 and 10966 respectively. The red curves show cumulative normal distributions corresponding to normal distributions all with a standard deviation of $0.062 \mathrm{DN} \mathrm{s}^{-1}$ pixel $^{-1}$, but different means $(5.934,5.829,5.722$ $\mathrm{DN} \mathrm{s}^{-1}$ pixel $^{-1}$, respectively). Also indicated are the $P_{z}$ values used for this work $\left(P_{z}=0.25\right.$, dark grey line) and that used by JBB $\left(P_{z}=0.45\right.$, light grey line). The data used for this analysis were from STEREO-A for a 10-day interval from 2009-06-11 to 2009-06-20. 


\subsubsection{Extraction of variation on small angular scales}

The next stage of processing was to separate the smooth distribution of zodiacal light from any small scale $\left(\sim 1^{\circ}\right)$ features arising from a ring-like stucture. Spatial models of the dust distribution, such as that of Kelsall et al. (1998) or Jones \& Rowan-Robinson (1993) can be used to predict surface brightnesses, but typically only to within a few

percent of observations: a model-based approach to the removal of zodiacal light is therefore unsuitable for detection of features fainter than this difference.

An alternative approach is to remove the contribution from the zodiacal light based on its observed spatial variability. This is expected to be smooth on scales exceeeding several degrees, and in particular, is expected to vary smoothly along directions parallel (or nearly parallel) to the plane of symmetry of the zodiacal cloud. Consequently, in order to locate features on small angular scales, we treated the map as a series of scans at constant ecliptic latitude. We then applied a filtering technique to each scan to remove variation on scales greater than about $6^{\circ}$.

Specifically, each scan was detrended using a two stage process. Firstly, a moving average surface brightness value, with the mean value being evaluated over a boxcar $13 \operatorname{bins}\left(\equiv 6.5^{\circ}\right)$ in extent is subtracted from each scan. This, in itself is insufficient to remove all large scale variation since the smooth background is known to have a surface brightness profile that is close to being a power law in $\lambda^{\prime}$ (Koutchmy \& Lamy 1985), and such a profile would leave a residual signal after boxcar filtering. In particular, if surface brightnesses, $S$, in a scan have the form

$$
S\left(\lambda^{\prime}\right)=k \lambda^{\prime-n}
$$

then the residual surface brightness obtained by applying a 13-point box-car filter is 
expected to be

$$
S_{\text {resid. }}\left(\lambda^{\prime}\right) \approx-7 k n(n+1)\left(\Delta \lambda^{\prime}\right)^{2} \lambda^{\prime-(n+2)}
$$

where $\Delta \lambda^{\prime}$ is the increment in helioecliptic latitude between adjacent bins in the scan. Typical values for the power law indicies $(n)$ were found to be $n \approx 1.8$ on the ecliptic plane, dropping to $n \approx 1.5$ at $|\beta|=8^{\circ}$.

For these data, the residual signal given by Equation 2 is typically greater than any signal due to the Venus ring. To account for this, JBB fitted each scan to a power law as in Equation 1, and the best fitting power law model was then detrended using the same moving average filter as applied to the data. A final scan was then produced by subtracting the detrended power law model from the detrended data scan. These scans were then used to construct a final image covering the coarse grid area. Because the window used for calcuation of the moving average is 13 bins wide, the original coarse grids are truncated by 6 bins $\left(\equiv 3.0^{\circ}\right)$ from each edge in helioecliptic longitude. This results in images with an extent $38.0^{\circ} \leq \lambda^{\prime} \leq 57.0^{\circ}$ (STEREO-A) or $-50.0^{\circ} \leq \lambda^{\prime} \leq-31.0^{\circ}$ (STEREO-B), with the extent in ecliptic latitude remaining $|\beta| \leq 8.5^{\circ}$. Overall then, images are produced by a process of coarse binning, selecting a probability cut as an estimator of the surface brightness of zodiacal light, and detrending using a box-car filter and correcting for an underlying power-law behaviour.

Images created using the above process are subject to systematic uncertainties due to the fact that the power-law form of the zodiacal light (Equation 1) is an approximation: the power-law index actually varies with $\lambda^{\prime}$. So for example, at $\beta=0.0^{\circ}$ for data set B080418, $n=-1.99$ at $\lambda^{\prime}=-33.25^{\circ}$, while $n=-1.84$ at $\lambda^{\prime}=-47.75^{\circ}$. Investigation of the effect of this variation revealed that it leads to systematic offsets in the final image, particularly for low values of $\left|\lambda^{\prime}\right|$. In a refinement to the detrending process, power-law fits to the scan were obtained for each point in a scan using a window 21 bins in width (i.e. 
on a scale of $10.5^{\circ}$ ). Where the scan point lay less than 10 bins from the end of the scan, the fitted power-law values at 10 bins from the relevant end of the scan were adopted. The modification to Equation 2 is that $n$ and $k$ then become slowly-varying functions of $\lambda^{\prime}$. The process is one of removing the local power-law approximation to the zodiacal light rather than a power-law that applies to the whole scan.

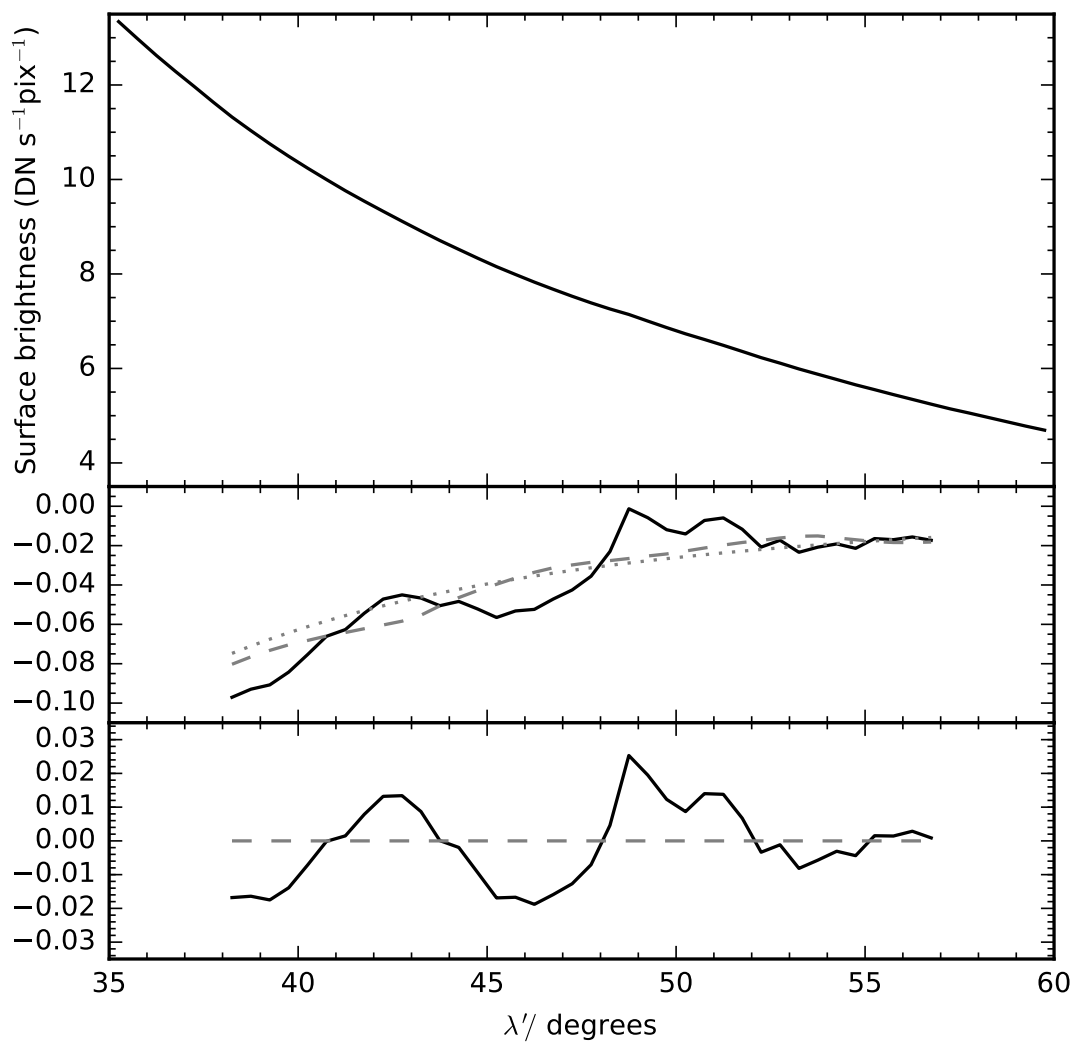

Fig. 4.- An example (using the data set A080616) of the steps involved in the image processing. Top panel: surface brightness in coarse grid bins centred in $\beta=0^{\circ}\left(P_{z}=0.25\right)$. Middle panel: solid line - the scan of the top panel after application of a box-car filter (13 bins); other lines show the residuals expected after applying the box-car filter to multiple (dashed-line) and single (dotted-line) power law fits to the scan. Bottom panel: the residual trace after removal of the expected residual of a multiple power-law fit from the scan in the middle panel. 


\subsubsection{An example processed image}

Figure 4 illustrates the stages in the image generation process using data set A080616. The top panel shows the surface brightness in the row of the coarse grid with $\beta=0^{\circ}$. The middle panel shows the effect of applying the 13-bin box-car filter to this scan, with the dashed line showing the effect of applying this filter to multiple power law fits to the data. For comparison, the dotted line shows the expected result of applying a 13-bin box-car filter

to a single power law fit to the entire scan (as given by Equation 2). The lower panel shows the residuals remaining after removing the expected signal from a multiple power-law fit to the scan.

The final image is then constructed using all of the scans from the coarse grid $|\beta|<8.5^{\circ}$. An example (again using the A080616 data set) is shown in Figure 5. Inspection of this image along $\beta=0^{\circ}$ shows the features that are visible in the residual trace in Figure 4 : there is a strong peak near $\lambda^{\prime}=49^{\circ}$, a trough that has a minimum at $\lambda^{\prime} \sim 46^{\circ}$, a weaker peak near $\lambda^{\prime}=42.5^{\circ}$, and another trough at $\lambda^{\prime}<40^{\circ}$. The importance of combining scans to create an image is highlighted by the distinctly different appearances of the peaks that were visible in the residual trace. The feature near $\lambda^{\prime}=49^{\circ}$ is also prominent in adjacent scans $\left(-2.5^{\circ}<\beta<+2.5^{\circ}\right)$ whereas the feature near $\lambda^{\prime}=42.5^{\circ}$ is only prominent at $\beta=0^{\circ}$. In the final image then, it is the feature at $\beta \sim 49^{\circ}$ that is prominent. It is for this reason that the mean surface brightness of several scans close to the ecliptic plane $\left(|\beta|<4.5^{\circ}\right)$ was adopted as a useful diagnostic for identifying the location of the ring feature (shown in the top panel of Figure 5).

The example image in Figure 5 also illustrates some of the limitations of the technique. The ring signal is weak: typical r.m.s. variation per bin is typically at the level of about $0.008 \mathrm{DN} \mathrm{s}^{-1}$ pixel $^{-1}$ (see Section 2.2.5) while the ring signal has a maximum amplitude of about $0.03 \mathrm{DN} \mathrm{s}^{-1}$ pixel $^{-1}$. There is always some residual signal from variation in the 
stellar background, such as the feature mentioned above at $\left(\lambda^{\prime}=42.5^{\circ}, \beta=0.0^{\circ}\right)$, but also at $\left(39.5^{\circ}, 6.0^{\circ}\right)$ and $\left(41.5^{\circ},-5.0^{\circ}\right)$. A characteristic of such features is that they are observed to move with the stellar background (i.e. by about $10^{\circ}$ from one data set to the next). This is in contrast to the ring feature, which is persistent at a fixed location on the image. (We also note that JBB illustrated the lack of such persistent features in a field offset from the orbit of Venus, see their Figure S1).

Another aspect of the image processing is that the observed position of the orbit of Venus changes over the ten-day interval over which the data were acquired. This is illustrated in Figure 5 by the plots of the orbit of Venus at the start and end times of the data set. Note particularly that the observed tangent to the orbit varies in $\beta$ by about $1^{\circ}$ over the 10-day data acquisition interval, and this is typical in many of the output images. A consequence of this is that any feature that is associated with the orbit of Venus will be blurred on a scale of about a degree in the direction perpendicular to the ecliptic plane, while there is no such effect in the longitudinal direction. This justifies our choice of coarse bin size, and in particular, the decision to have bins of extent $1.0^{\circ}$ in $\beta$, while their extent in $\lambda^{\prime}$ is smaller, at $0.5^{\circ}$.

\subsubsection{Sensitivity of image processing to choice of $P_{z}$}

It was noted above that the surface brightness of zodical light was determined (to within a constant offset value) by selecting a value from the cumulative distribution function of pixels in a bin corresponding to a probability $P_{z}=0.25$. Since this is an important aspect of the image processing technique, it was investigated in depth. We note that JBB adopted a probability value of $P_{z}=0.45$ as the estimator for the surface brightness of zodiacal light based on modelling the distribution function for L1 image pixels. Justification for this approach can be seen from Figure 3: this value of $P_{z}$ provides an estimate of surface 
(a)

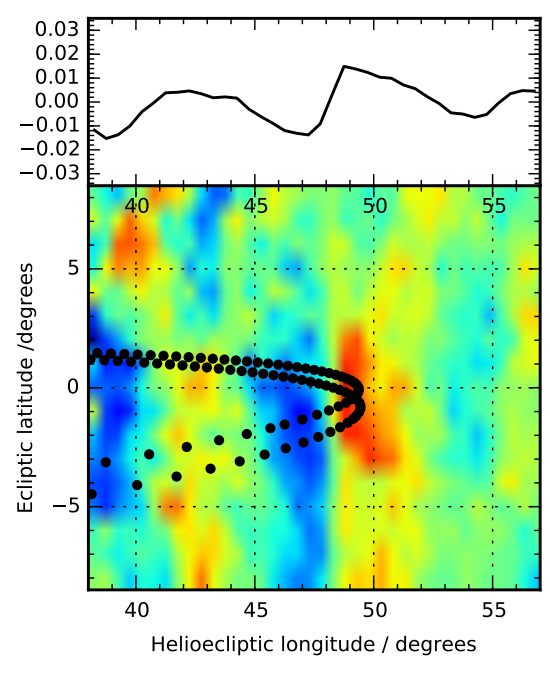

Surface brightness (DN s${ }^{-1}$ pix $\left.^{-1}\right)$ (b)

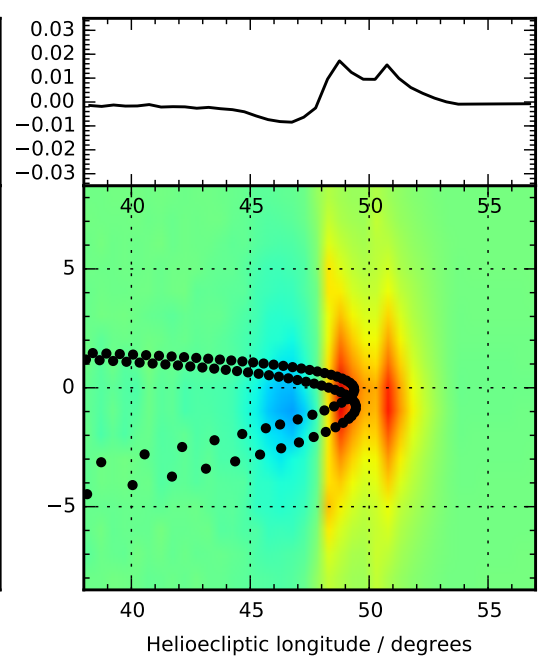

(c)

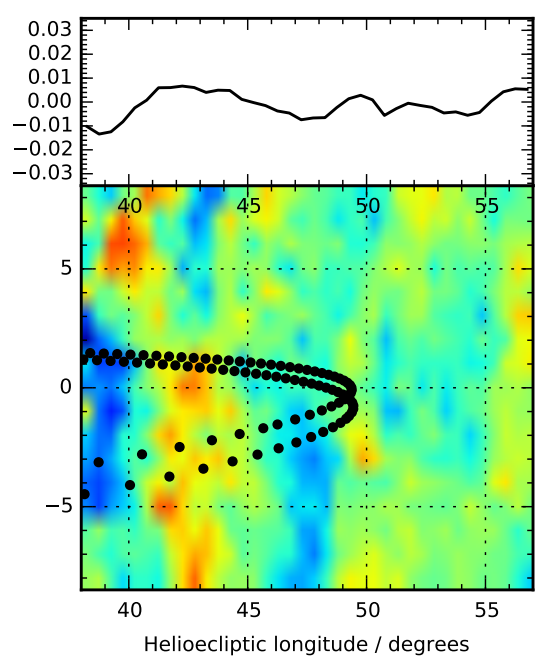

Fig. 5.- (a) An example (using the data set A080616) of an image produced using the detrending technique described in the text. The black dots on the image show the orbit of Venus at the start and end times of the data set (i.e. 2008-06-16 00:00 UTC and 2008-06-26 00:00 UTC respectively). The trace above the image shows the mean surface brightness over $|\beta|<4.5^{\circ}$ in units of $\mathrm{DN} \mathrm{s}^{-1}$ pixel $^{-1}$. (b) The synthetic image and the corresponding mean surface brightness scan $\left(|\beta|<4.5^{\circ}\right)$ for the best-fitting two-step ring model (as described in the text) to the image. (c) The residual image and corresponding mean surface brightness scan $\left(|\beta|<4.5^{\circ}\right)$ obtained by subtraction of the model in (b) from the data in (a). 
brightness (as measured from the black curves) that is close to the means of the symmetric components of the distributions (red curves). Since any constant offset values are removed by the processing technique, it may be the case that lower values of $P_{z}$ may result in images with less residual noise from the stellar background.

This was investigated by processing the images using a range of values of $P_{z}$. For each of the resultant images we calculated the root mean square variation in surface brightness in the image bins. The data sets used were B080607 and B080617. These data were presented by JBB to show the absence of systematic features in regions away from the orbit of Venus, with images covering $-67.0^{\circ} \leq \lambda^{\prime} \leq-48.0^{\circ}$ (see JBB, Supplementary Materials Figure S1). Figure 6 shows r.m.s. variation between bin values (over the entire image) as $P_{z}$ is varied: there is a shallow minimum over the range $0.05<P_{z}<0.30$ where the r.m.s. variation is about $0.008 \mathrm{DN} \mathrm{s}^{-1}$ pixel $^{-1}$. Our choice of a value of $P_{z}=0.25$ is therefore expected to be an improvement over the work presented by JBB since the residual nose levels in images should be reduced.

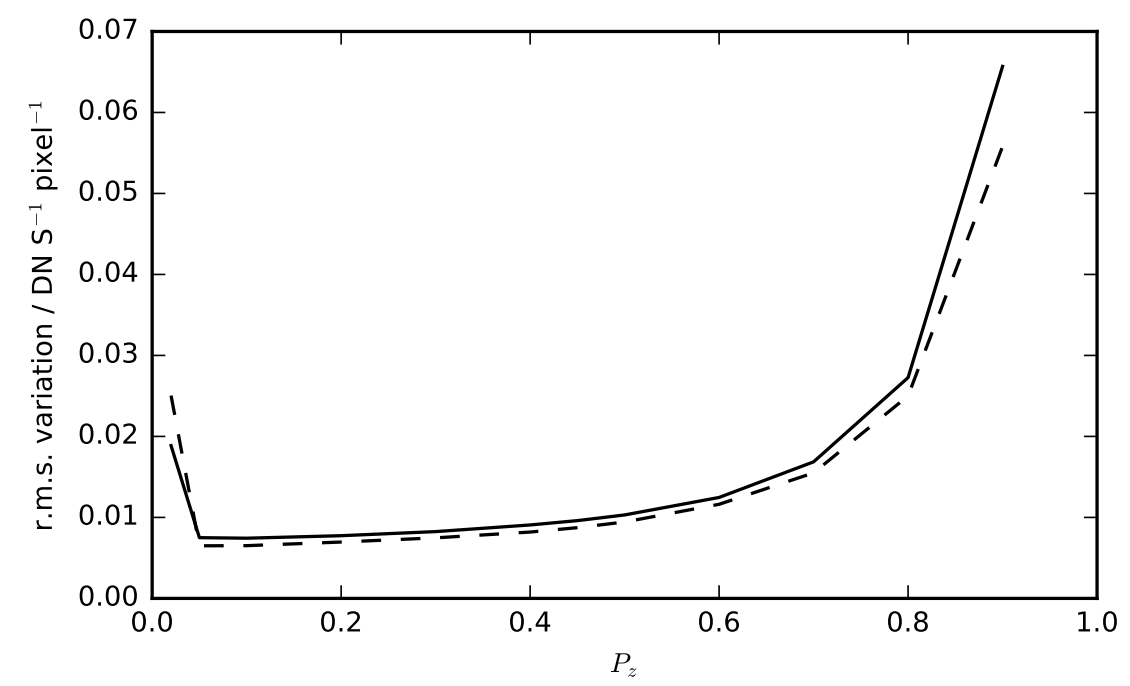

Fig. 6. - The variation in root mean square residual surface brightness with probability cut $P_{z}$. The data sets are B080607 (solid line) and B080617 (dashed line), using a field that is offset from the location of the orbit of Venus (see text for details). 


\section{3. $\quad$ Ring modelling}

In order to interpret the processed images, we invoked a spatial model for the dust distribution and generated synthetic images that could be compared against observation. Synthetic images were generated by calculating the expected surface brightness at the centres of the coarse grid bins described in Section 2.2. To account for the changes in viewing geometry that occur in the 10-day duration of a real data set, the synthetic data used spacecraft positions from the real data set. However, rather than simulating every L1 image in a set, it was found to be sufficient to use only 1 in every 5 images as a basis for modelling the changes in viewing geometry: changes on timescales of less than 10 hours result in differences in the output model that are not detectable at the spatial resolution of the coarse grid.

For each bin centre and viewing location, surface brightnesses were calculated by integration along a line of sight of total length 2 AU, with a step-length of 0.005 AU. The dust density at any given point was specified by a geometric model that is fixed with respect to a (non-rotating) heliocentric frame of reference. Two particular models were used in this work: the fan model of Kelsall et al. (1998), albeit modified to be centred on the Sun, and, a two-step ring model as adopted by JBB. In calculating the contribution from scattered light at any point along a line of sight, we adopted the optical scattering function of Hong (1985).

As in JBB, the purpose of using the Kelsall et al. (1998) fan model in this analysis is to provide a reference density of the IPD cloud at the orbit of Venus that is linked to the observed surface brightness of the zodiacal light. This model is expected to provide a good representation of the radial dependence of dust density (see e.g. Rowan-Robinson \& May 2013) and this is an important factor in determining the density at the orbit of Venus. The model includes a scaling factor to relate observed surface brightness (in instrumental 
units) to model dust density. This scaling factor was determined from the observed surface brightnesses in the STEREO-A data as follows. The mean surface brightness over the coarse grid area was determined for nine data sets (identified in Table 1). Simulations of surface brightnesses from the fan model were made for the coarse grid area for these observations. For each simulation the scaling factor of the model was set so that the model and observed mean surface brightness matched. It is noted that for individual data sets, systematic differences between the model and observed surface brightnesses result in a standard deviation of about 10 percent. For the nine observations used in this process, the scaling factors agreed to within \pm 1.5 percent, and with no obvious trend in the normalisation factors over time. This is as expected given the very stable response of the HI-2 instruments reported by Tappin et al. (2015). We note that in this work, the scaling factor is 4 percent greater than the value adopted by JBB.

The corresponding normalisation factor for the model HI-2B data was found by applying the calibration corrections of Tappin et al. (2015) to the HI-2A normalisation factor. Tappin et al. (2015) note that HI-2B is the more sensitive instrument, and the HI-2B normalisation factor would be expected to be $1.103(=1 / 0.907)$ times that of HI-2A.

In fitting a model for the ring to an image, the model was used to create a synthetic image that was subject to the same box-car filtering as applied to the actual data. Since the ring model was not used in conjunction with a model for the IPD cloud, the detrending process for the synthetic data did not include the removal of the power-law contribution (Equation 1) to the surface brightness. Parameter fitting was carried out using a $\chi^{2}$ minimisation routine to find the synthetic image that best matches the data. In this process only part of the image was used: $45.0^{\circ}<\lambda^{\prime}<55.0^{\circ}$ for HI-2A, and $-39.0^{\circ}>\lambda^{\prime}<-49.0^{\circ}$ for HI-2B. For each image an estimate of the standard deviation associated with binto-bin variation was made using parts of the image located away from the Venus ring 
(HI-2A: $44.0^{\circ}<\lambda^{\prime}<45.0^{\circ}$ and $55.0^{\circ}<\lambda^{\prime}<56.0^{\circ}$; HI-2B: $-38.0^{\circ}>\lambda^{\prime}>39.0^{\circ}$ and $\left.-49.0^{\circ}>\lambda^{\prime}>-50.0^{\circ}\right)$

An example of the fitting process, again as applied to A080616, is shown in Figure 5b. The details of the model are described in Section 3, but it should be noted that only two parameters of the model were fitted to an individual image, these being the densities at the

first and second step, with the other ring parameters being determined from the ensemble of images. Figure 5c shows the residual image obtained once the best-fitting model is removed from the data.

\section{Results}

The techniques described above were employed to create a set of all usable images from HI-2A and HI-2B covering the period 2007-2014. These images were then used to investigate two physical aspects of the Venus ring: its orientation, and its azimuthal structure.

\subsection{Images}

The data sets listed in Tables 1 and 2 were processed to create images which were visually inspected to check for the presence of ghost images, high noise levels, or large systematic offsets close to the ecliptic plane. This process led to the rejection of 14 data sets (2 from HI-2A and 12 from HI-2B)

A check was also made for the presence of optically bright nebulae from the catalogue of Lynds (1965). This catalogue covers all areas of the celestial sphere that are part of this study. While the majority of these Lynds bright nebulae (LBN) are excluded by our data selection criterion that excludes the galactic plane, there are $18 \mathrm{LBN}$ at galactic latitudes 
$|b|>30^{\circ}$ that lie within $8.5^{\circ}$ of the ecliptic plane, and which could potentially affect our images. The positions of these LBN over the 10-day integration periods were overlaid on images to check for contamination. Figure 7 shows an example of potential contamination, with eleven LBN present in the image. Background objects typically move by about $10^{\circ}$ over the image integration period. The filtering process removes features on angular scales exceeding $6.5^{\circ}$, but there is likely to be a residual sigal near the start and end positions. This form of this residual depends on the extent and shape of the object, and would be difficult to model accurately. Consequently we rejected any images in which the tracks of LBN could give rise to a spurious signal. This led to the further rejection of seven images (4 from STEREO-A and 3 from STEREO-B) all of which had centres in the range of ecliptic longitude $29^{\circ}<\lambda<50^{\circ}$ (corresponding to a region between $(\alpha, \delta)=\left(1^{\mathrm{h}_{48}} \mathrm{~m},+11^{\circ}\right.$ ) and

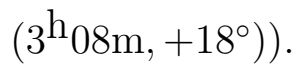

Tables 1 and 2 summarise all data sets that met the original selection criteria and include notes on any that were rejected at this stage. The remaining data sets (46 from HI-2A and 19 from HI-2B) form the basis of the study of the Venus ring presented here. The full set of these images is available online (Supplementary Material - processed images).

An example set of images is shown in Figure 8, showing all images from HI-2A data sets from A130201 to A130831. These images show some of the features of the Venus ring as reported by JBB (but in data not reported by JBB) and illustrate some of the limitations of the data. Data sets A130201 to A130402 (Figures 8a to g) show a relatively strong (peak height of $0.030 \mathrm{DN} \mathrm{s}^{-1}$ pixel $^{-1}$ ) that is slightly interior to the orbit of Venus. (This behaviour is similar to the sequence comprising A090609, A090629, A090719 presented by JBB.) There is a break in the observation sequence (due to the presence of the Galactic plane near the mapping region) and when observations recommence with A130722, the peak is now exterior to the orbit of Venus (similar to observation A081112 reported by 


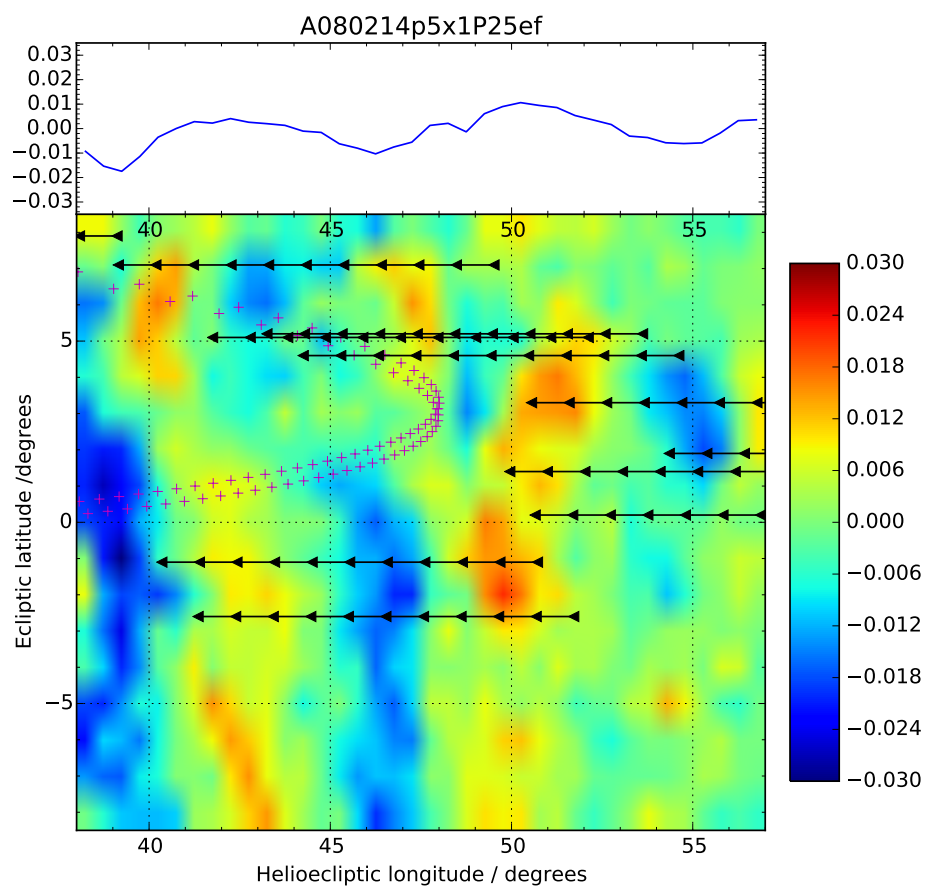

Fig. 7.- An example of an image that is potentially contaminated by optical emission from Lynds (1965) bright nebulae. The tracks of such nebulae over the 10-day integration period of the data are indicated by solid black lines, with arrow markers at one-day intervals. Because of the potential contamination, this data set (A080214) is rejected from the final analysis. 
JBB). There are however, limitations to the data. Although the maps are generated to minimise the variation due to the stellar background, the images include fluctuations that could distort any signal from a dust ring at Venus. It is evident from a sequence such as this that although there is reasonable agreement between the position of the strong feature and the tangent to orbit of Venus, the correspondence is not perfect. Futhermore, in cases where the ring signal is weak (Figures $8 \mathrm{k}$ and $\mathrm{l}$ ) some caution is needed in interpreting features in the images.

\subsection{Ring orientation}

One of the key questions about the observed ring feature is whether its orientation matches that of the orbit of Venus. The processed images described above provide a means to test this. We adopted the following method to locate regions of enhanced surface brightness and to estimate the central position of any group of bins that constitute such a region.

Two surface brightness thresholds, $S_{1}$ and $S_{2}$ are defined (with $S_{1}<S_{2}$ ). $S_{1}$ is used to determine whether a bin in the image may be part of a group and $S_{2}$ is used to define when a group is to be included. A box-car smoothing with length 3 bins is applied to the image and all bins with surface brightness $S_{i, j}>S_{1}$ are identified as possible enhancement bins. These bins are then grouped together to form a contiguous group. For two bins to be considered to be contiguous, they must both satisfy the surface brightness threshold and be adjacent in the sense that they share a common bin edge (i.e. bins that touch only at corner points are not considered contiguous). This is repeated for all bins to identify all contiguous groups noting there may be more than one potential group identified. A group

must contain a minimum of 4 bins, and have a maximum bin value of $S_{\max } \geq S_{2}$ in the unsmoothed image. Any group that does not meet these criteria is eliminated. 


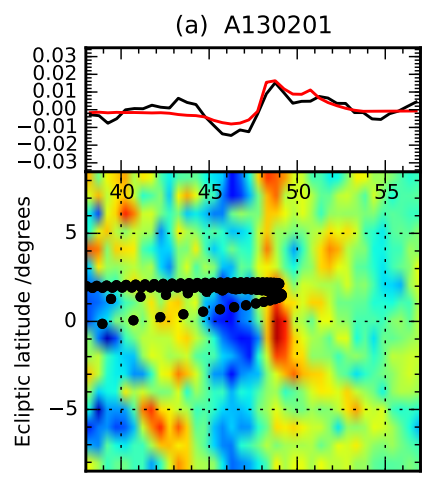

(e) A130313

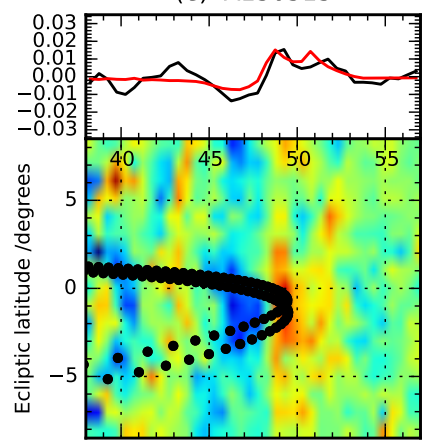

(i) $\mathrm{A} 130801$

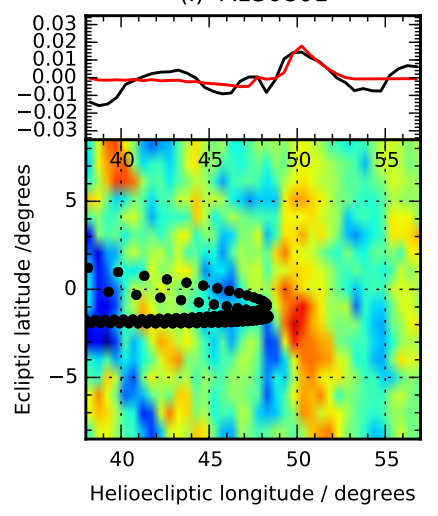

Surface brightness (DN s${ }^{-1}$ pix $\left.^{-1}\right)$ (b) A130211

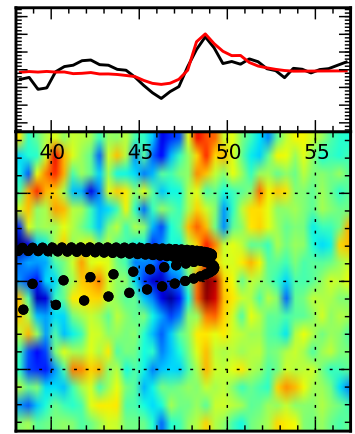

(f) $\mathrm{A} 130323$

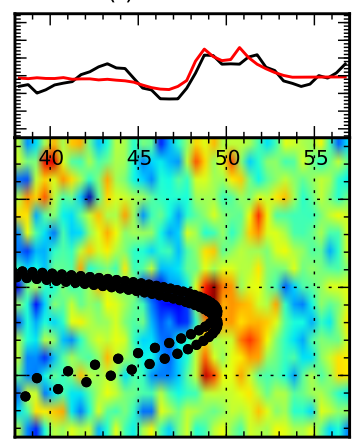

(j) A130811

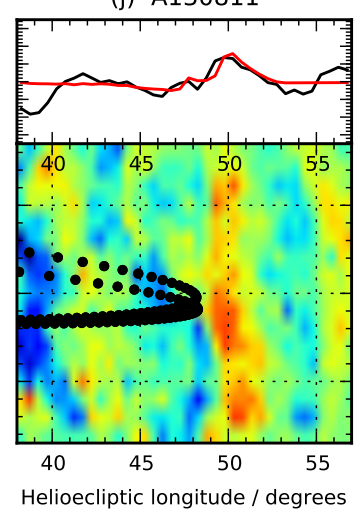

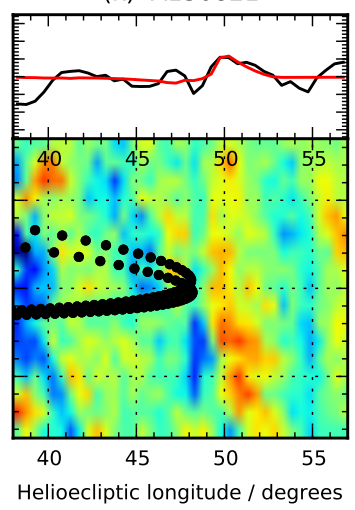

(d) A130303

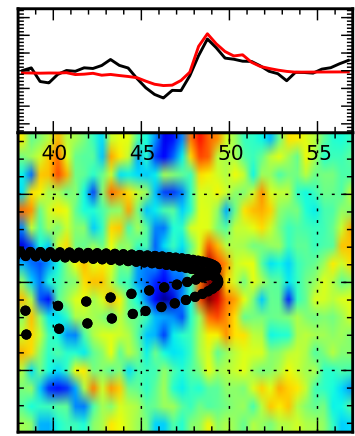

(g) A130402

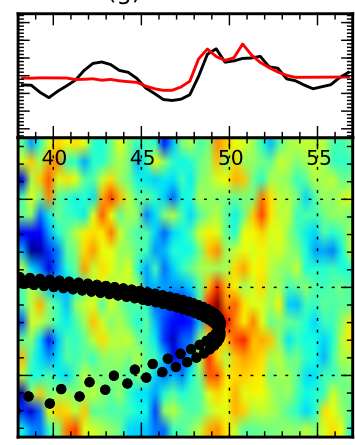

(h) A130722

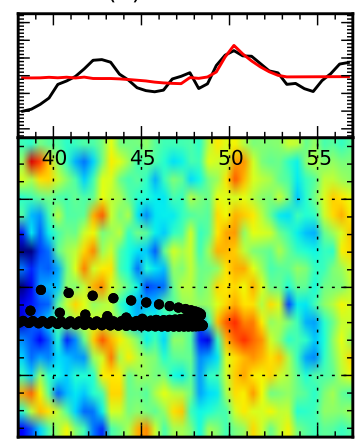

(I) A130831

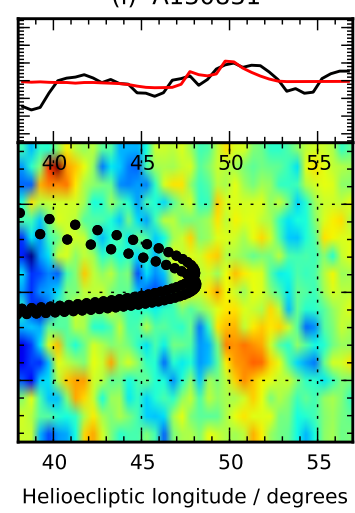

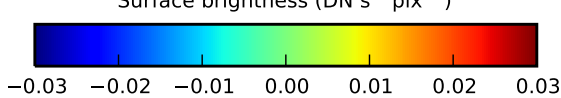

Fig. 8. - Residual surface brightness maps from HI-2A data obtained between February and early September 2013 (all sets from A130201 to A130831). The traces above each image show the mean surface brightness over $|\beta|<4.5^{\circ}$ in units of $\mathrm{DN} \mathrm{s}^{-1}$ pixel $^{-1}$, with data in black and best-fit model (see Section 3.3) in red. 
For each remaining group, a central position is calculated using a weighting based on the surface brightness above the threshold (i.e. analogously to a centre of mass). The weighting is defined by

$$
w_{n}=\left(\max \left(\left(S_{n}-S_{1}\right), 0\right)^{2}\right.
$$

and the estimator of the position of the central point of the group is defined by

$$
\begin{aligned}
\lambda_{c}^{\prime} & =\frac{\sum w_{n} \lambda_{n}^{\prime}}{\sum w_{n}} \\
\beta_{c} & =\frac{\sum w_{n} \beta_{n}}{\sum w_{n}}
\end{aligned}
$$

where the summations are over all bins in a contiguous group.

The following threshold values were taken, $S_{1}=0.007 \mathrm{DN} \mathrm{s}^{-1}$ pixel $^{-1}$ and $S_{2}=0.009 \mathrm{DN} \mathrm{s}^{-1}$ pixel $^{-1}$. These were chosen as they typically picked out significant enhancements in the maps without creating too many fragmented groups. In cases where multiple groups were identified in an image, the most prominent group was selected by eye as being representative of the ring feature.

Of particular interest is the way in which the ecliptic latitude, $\beta_{c}$, of the ring feature varies with the ecliptic longitude of the tangent to the ring $\lambda_{T}$ : this should indicate the inclination of the ring as well as the ecliptic longitude of its ascending node. Because observations are made over a range of distances from the Sun (and hence a range of distances from the tangent point to a ring at Venus), it is necessary to correct the observed ecliptic latitudes so that they all correspond to an observation made from a standard distance. Hence we correct the A and B data to correspond to a viewing position at 1.0 AU from the Sun. The corrected ecliptic latitude is $\beta_{c, 1.0}=\tan ^{-1}\left(r \tan \beta_{c}\right)$, where $r$ is the 
distance in the ecliptic plane from the Sun to the relevant spacecraft (expressed in AU).

The upper panel of Figure 9 shows how $\beta_{c, 1.0}$ varies with $\lambda_{T}$. These data were fitted to a model of a ring with radius equal to the semi-major axis of the orbit of Venus, but with inclination $i_{\text {ring }}$ and longitude of ascending node $\Omega_{\text {ring }}$ to be determined. The peak positions were all assumed to have the same measurement uncertaintity. While this measurement uncertainty is unknown, it is unlikely to be less than the angular resolution of the image in the latitudinal direction $\left(1.0^{\circ}\right)$, and given the observed spread in values is very unlikely to exceed $\left(2.5^{\circ}\right)$. We therefore adopt an indicative uncertainty of $1.5^{\circ}$.

Parameter values were found by $\chi^{2}$ minimisation, with best-fit parameter values of $i_{\text {ring }}=2.1^{\circ}$ and $\Omega_{\text {ring }}=68.5^{\circ}\left(\chi^{2}=35.0,63\right.$ degrees of freedom $)$. The $\chi^{2}$ surface for this model is shown in the lower panel of Figure 9 : note that the parameter values corresponding to the orbit of Venus (i.e. $i_{\text {ring }}=3.39^{\circ}$ and $\Omega_{\text {ring }} 76.68^{\circ}$ ) lie just outside the 99.73 percent confidence interval, indicating that the ring orientation differs from the orbital plane of Venus. Some caution has to be expressed at the significance of of this result. Firstly, the uncertainty in the peak positions is not well determined. If the uncertainty is higher than $1.5^{\circ}$ then the Venus ring parameter values will lie within a lower confidence interval: for instance, with a measurement uncertainty of $2.5^{\circ}$ the Venus orbit values are excluded only at the 95.4 confidence level. Secondly, we note that the data near the extreme values of peak position (i.e. $\lambda_{T} \sim 170^{\circ}$ and $\lambda_{T} \sim 350^{\circ}$ ), which would help to constrain the fitted parameter values, cannot be observed because these lines of sight are close to the galactic plane. Overall however, we tentatively conclude that the dust ring has a different inclination and longitude of ascending node to that of the orbit of Venus. 

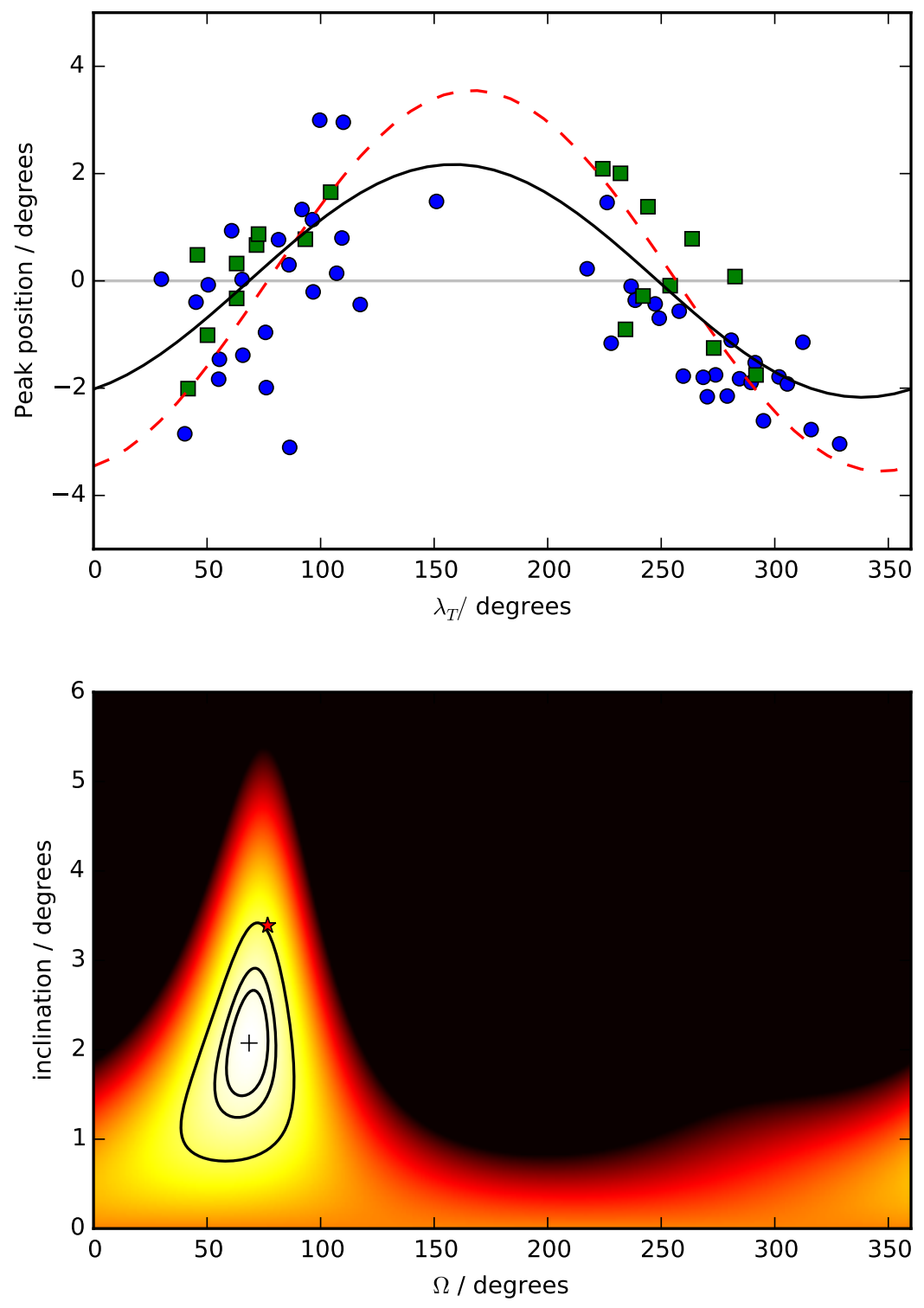

Fig. 9.- (Upper panel) The ecliptic latitude of the mid-position of bright features in images from HI-2A (blue circles) and HI-2B (green squares) data against the longitude of the tangent point to the line-of-sight of a ring at the orbit of Venus. These ecliptic latitudes have been adjusted to correspond to a viewing location at 1.0 AU from the Sun. The expected variation of the ecliptic latitude of the tangent point to a circular ring with $\Omega_{\text {ring }}=68.5^{\circ}$ and $i_{\text {ring }}=2.1^{\circ}$ is shown by the solid black line. Also shown is the expected variation for a ring aligned with the orbit of Venus (dashed red line). (Lower panel) The $\chi^{2}$ surface for the two ring parameters $\Omega_{\text {ring }}$ and $i_{\text {ring. }}$. The cross at $\Omega_{\text {ring }}=68.5^{\circ}$ and $i_{\text {ring }}=2.1^{\circ}$ shows the minimum at $\chi^{2}=35.0$ (63 degrees of freedom). Confidence intervals are shown at the 68.3, 95.4 and 99.73 percent levels. The ring parameter values of the orbit of Venus are shown by the red star. The shading ranges from white at $\chi^{2}=35$ to black at $\chi^{2}=105$. 


\subsection{Azimuthal structure of the ring}

The approach taken to studying the azimuthal structure of the ring is based on the idea that any observation provides information about the ring in the vicinity of its tangent point to the line-of-sight. If the ring has its origin in resonant trapping of dust on exterior orbits, then it might be expected that the distribution of dust should remain fixed with respect to a coordinate system that rotates with the mean motion of Venus (because Venus should correspond to the Case I type resonance described by Kuchner \& Holman 2003). It is therefore appropriate to determine how the properties near the tangent point to the ring

vary with the co-rotating longitude $\theta$ (see Figure 1). The other coordinates in this rotating coordinate system are the radial distance, $r$, from the Sun, and a vertical distance, $z$, from the plane of the ring. The orientation of this ring is as determined above $\left(\Omega_{\text {ring }}=68.5^{\circ}\right.$ and $i_{\text {ring }}=2.1^{\circ}$.

As in JBB, the model we adopt for the ring density has two radial steps in density and an exponential drop off away from the plane of the ring.

$$
n(r, \theta, z)=n_{\theta}(r) e^{-|z| / \sigma_{z}}
$$

where

$$
n_{\theta}(r)= \begin{cases}0 & \text { if } r<r_{0} \\ n_{0, \theta} & \text { if } r_{0}<r<r_{1} \\ n_{1, \theta} & \text { if } r>r_{1}\end{cases}
$$

Every image that is fitted to this model potentially provides information on the parameters $r_{0}, r_{1}, \sigma_{z}, n_{0, \theta}$ and $n_{1, \theta}$. As we are at the limits of the sensitivity of the observations, we cannot obtain firm constraints on all five parameters for each image, so we adopt an approach of fixing some model parameters based on the weighted mean of best fit values from the whole set of images. 
As in JBB, we make the assumption that $\sigma_{z}$ does not vary between observations. We conducted a first set of fits to determine $\sigma_{z}$ values for each image, but then determined the weighted mean value to be applied in a second iteration of fitting. The weighted mean value obtained was $\sigma_{z}=0.0518 \mathrm{AU}$, noting that this is close to the value of $0.05478 \mathrm{AU}$ found by JBB using a much smaller data set. Inspection of the values of $r_{0}$ and $r_{1}$ that were obtained in the second iteration revealed no clear variation in these values with $\theta$, thus justifying a decision to fix these parameters also. The weighted mean values obtained and which were subsequently used were $r_{0}=0.71524 \mathrm{AU}$ and $r_{1}=0.73917 \mathrm{AU}$ (noting, as in JBB, that these locations lie either side of the semi-major axis of the orbit of Venus). In the third iteration of fitting, the only free parameters are $n_{0, \theta}$ and $n_{1, \theta}$, i.e. the in-plane densities at the first and second steps respectively.

An illustration of variation in the ring density at the tangent point is shown in Figure 10. This shows the best fit to four data sets, representing all combinations of high and low values of $n_{0, \theta}$ and $n_{1, \theta}$.

The best-fit values of $n_{0, \theta}$ and $n_{1, \theta}$ are shown in Figure 11 for all usable data from the interval 2007-2014 (a table of these values is available online, see Supplementary Material - density fitting). This plot constitutes a description of how the densities of the inner and outer steps in the model vary around the ring (i.e. with $\theta$ ). In interpreting such a plot, the following points should be noted. The origin of the co-rotating longitude $(\theta)$ is, to a good approximation, coincident with Venus, and negative values of $\theta$ correspond to locations trailing behind the planet in its orbit. Note that no images are obtainable for $|\theta|<38^{\circ}$ because the detrending method fails if Venus is present in the field of view. It should also be noted that the densities $n_{0, \theta}$ and $n_{1, \theta}$ are expressed in terms of the density of the Kelsall et al. (1998) fan model (as described above) at a heliocentric distance corresponding to the semi-major axis of the orbit of Venus (a value we refer to as $n_{\mathrm{fan}, V}$ ). Finally, note that the 

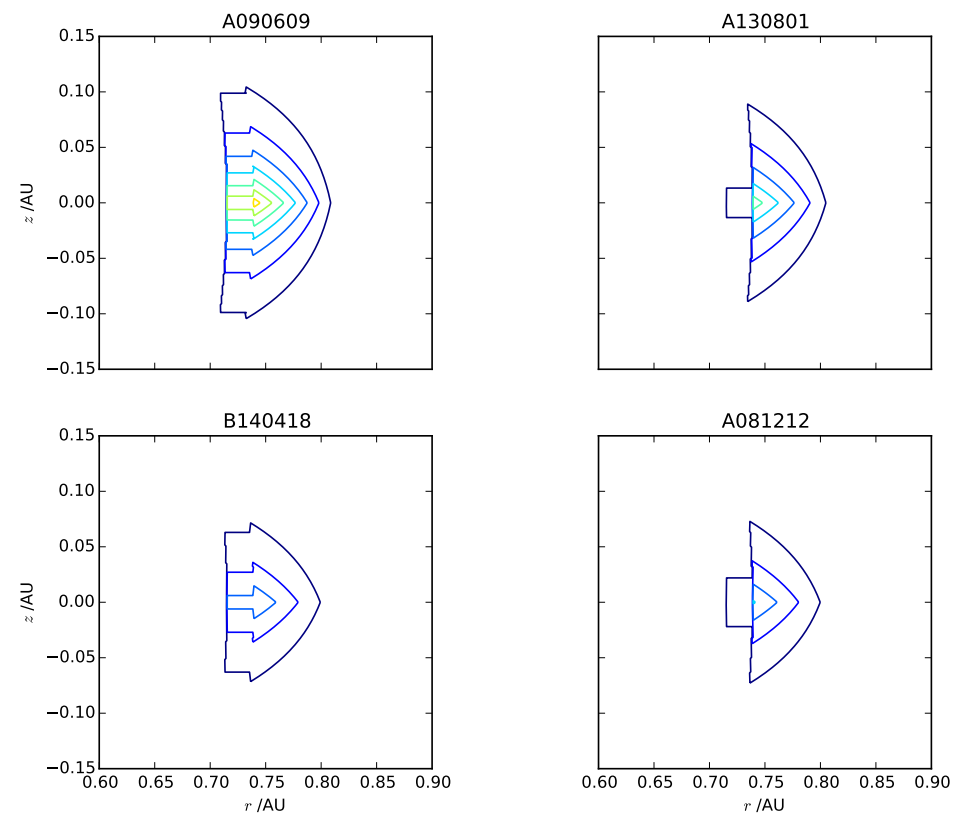

Fig. 10. - Examples of the ring model that illustrate the implied density in the verticalradial plane for all combinations of high and low values of $n_{0, \theta}$ and $n_{1, \theta}$. Top left: (A090609) high- $n_{0, \theta}$, high- $n_{1, \theta}$. Top right (A130801) low- $n_{0, \theta}$, high- $n_{1, \theta}$. Bottom left (B140418) high$n_{0, \theta}$, low- $n_{1, \theta}$. Bottom right (A081212) low- $n_{0, \theta}$, low- $n_{1, \theta}$. The contour interval is 1 percent of $n \mathrm{fan}, \mathrm{V}$. Note that the density at radial distances $r$ greater than $r_{1}$ shown here have a linear decline (over a distance of $0.08 \mathrm{AU}$ ) that is not part of the fitted model. This has been done to meet the expectation that the ring density must drop with increasing $r$, but this cannot be detected because of the filtering technique used. The spatial scale is given in AU, and the ring is shown with respect to its plane of symmetry. 

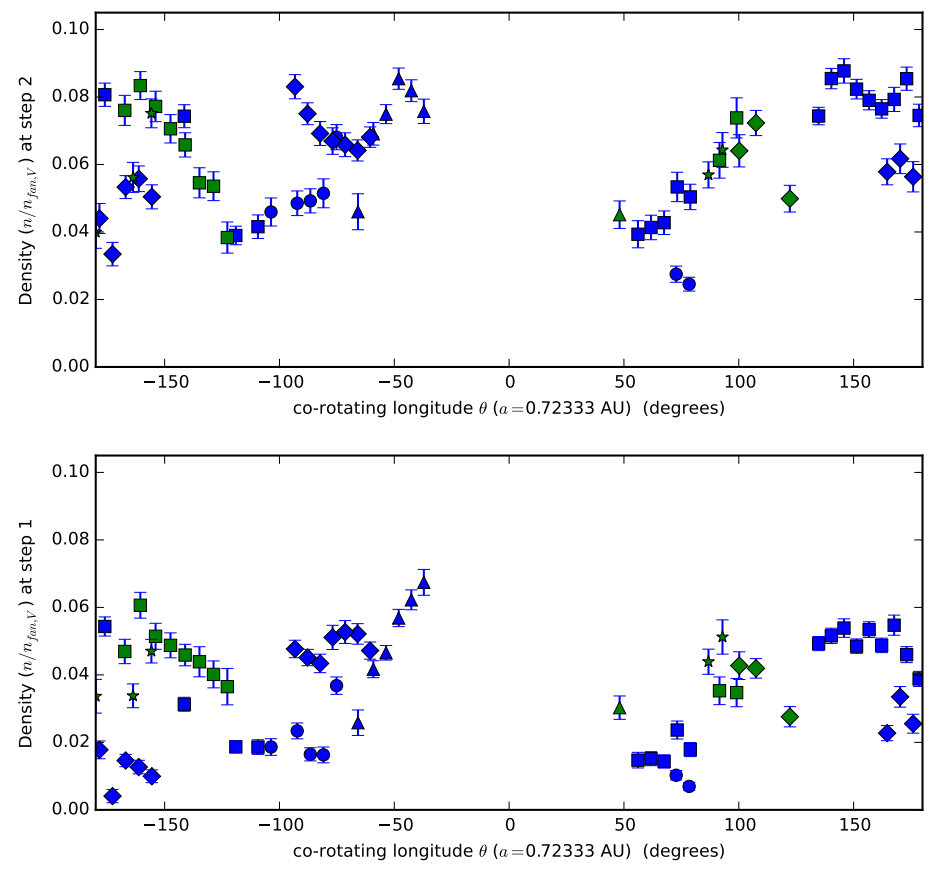

Fig. 11. - Fitted values of $n_{0, \theta}$ (lower panel) and $n_{1, \theta}$ (upper panel) against co-rotating longitude $\theta$. HI-2A data are in blue, HI-2B data are in green. Key: o-2007, $\square-2008, \triangle-2009$, $\diamond-2013, \star-2014$. 
density $n_{1, \theta}$ represents the total density at the second step and is therefore given by $n_{0, \theta}$ plus the increase in density at the second step.

It is clear that Figure 11 shows significant discrepancies between density values returned at similar values of $\theta$. However, on closer inspection, it appears that many of the more significant discrepancies are between observations that are widely separated in time. For example for $\theta \sim-170^{\circ}$ values of $n_{0, \theta}$ obtained in 2008 are typically about $0.05 n_{\mathrm{fan}, V}$, whereas in 2013 they are less than $0.02 n_{\mathrm{fan}, V}$. Another example is that at $\theta \sim-100^{\circ}$, in $2007 n_{1, \theta}<0.05 n_{\mathrm{fan}, V}$ while in $2013 n_{1, \theta} \sim 0.08 n_{\mathrm{fan}, V}$. A more coherent picture of the ring density emerges if the data are split into two epochs; firstly, the data from 2007-2009 (Figure 12), and secondly from 2013-2014 (Figure 13).
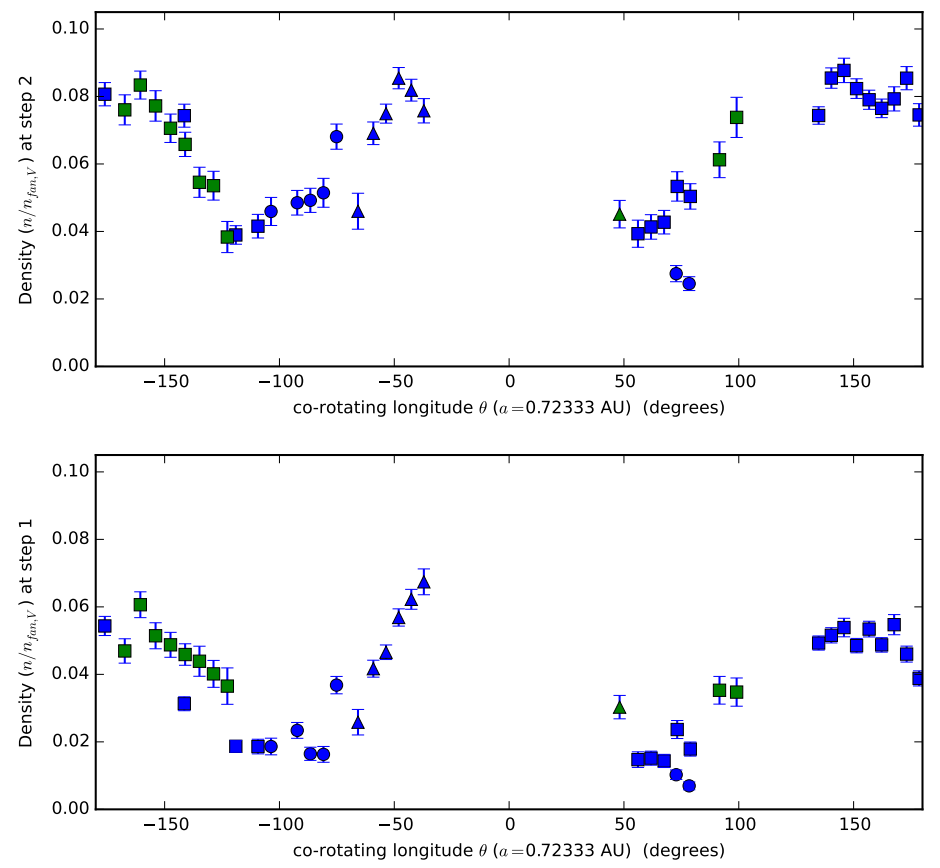

Fig. 12.- Fitted values of $n_{0, \theta}$ (lower panel) and $n_{1, \theta}$ (upper panel) against co-rotatating longitude $\theta$. HI-2A data are in blue, HI-2B data are in green. Key: o-2007, $\square-2008, \triangle-2009$.

The data set for 2007-9 (Figure 12) shows better coverage over the range of $\theta$ than the 2013-14 data. There is fairly good agreement between values obtained from the two 

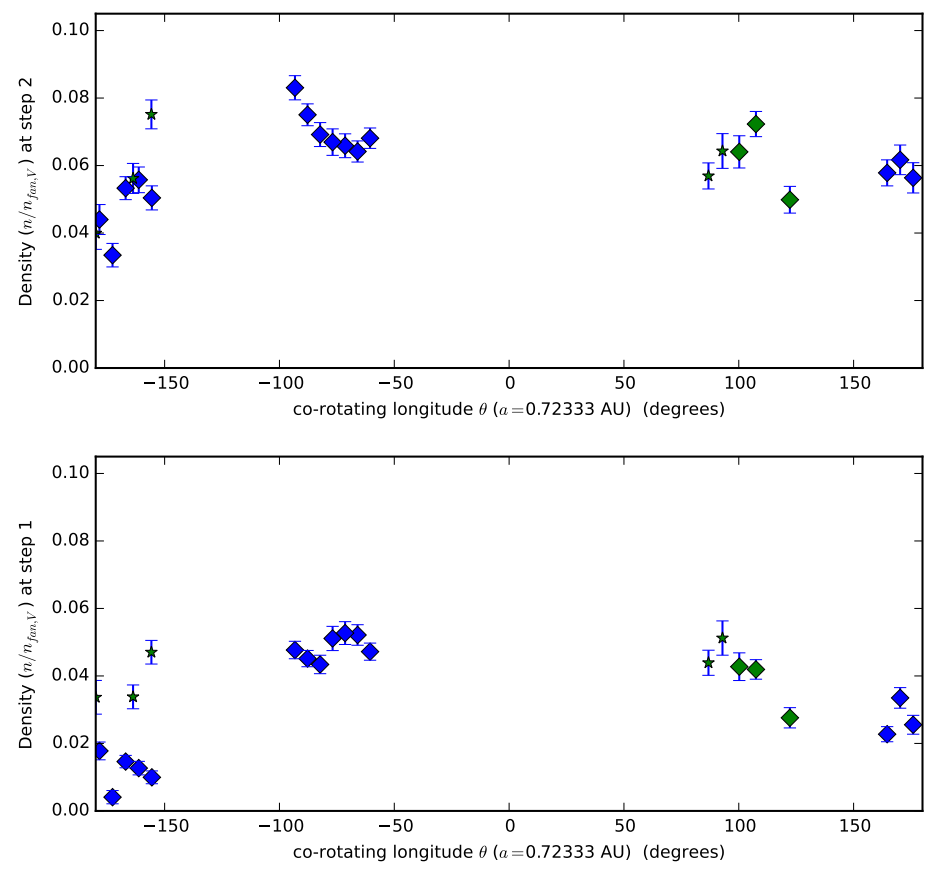

Fig. 13.- Fitted values of $n_{0, \theta}$ (lower panel) and $n_{1, \theta}$ (upper panel) against co-rotating longitude $\theta$. HI-2A data are in blue, HI-2B data are in green. Key: $\diamond-2013, \star-2014$.

instruments at similar values of $\theta$ (see $-180^{\circ}<\theta<-130^{\circ}$ in Figure 12). There still remain some discrepancies between the values of density obtained at similar values of $\theta$, but overall there is better agreement than in Figure 11. Significantly, there is reasonable agreement between data acquired in 2007 and 2009, which indicates that the pattern of density variation is approximately constant in the frame of reference that co-rotates with the mean motion of Venus.

The pattern of density enhancement in the 2007-9 data shows $n_{0, \theta}$ and $n_{1, \theta}$ varing considerably but in a rather smooth fashion. Following the pattern from $\theta=0^{\circ}$ backwards along the orbit, the following features can be described. There is a peak in $n_{0, \theta}$ and $n_{1, \theta}$ $\left(\sim 0.07\right.$ and $0.08 n_{\mathrm{fan}, V}$ respectively) that trails Venus $\left(\theta \sim-40^{\circ}\right)$. Densities then decline to low values $\left(n_{0, \theta} \sim 0.02\right.$ and $\left.n_{1, \theta} \sim 0.04 n_{\text {fan }, V}\right)$ near $\theta \sim-100^{\circ}$, before rising again at $\theta \sim-140^{\circ}$, to $n_{0, \theta} \sim 0.05$ and $n_{1, \theta} \sim 0.08 n_{\text {fan }, V}$, before declining at $\theta \sim+50^{\circ}$ to low values 
again $\left(n_{0, \theta} \sim 0.02\right.$ and $\left.n_{1, \theta} \sim 0.04 n_{\mathrm{fan}, V}\right)$.

The data set for 2013-4 is far less complete, but shows the following pattern (again starting from $\theta \sim 0^{\circ}$ and moving backwards along the orbit). At $\theta \sim-60^{\circ}$ to $-100^{\circ}$ there are relatively high dust densities $\left(n_{0, \theta} \sim 0.05\right.$ and $\left.n_{1, \theta} \sim 0.07 n_{\mathrm{fan}, V}\right)$. Near $\theta \sim-160^{\circ}$, there are conflicting data: in 2013 (HI-2A data) $n_{0, \theta}<0.02 n_{\mathrm{fan}, V}$ while in 2014 (HI-2B data) $n_{0, \theta} \sim 0.04 n_{\mathrm{fan}, V}$. At $\theta \sim+170^{\circ}, n_{0, \theta} \sim 0.03$ and $n_{1, \theta} \sim 0.05 n_{\mathrm{fan}, V}$, and by $\theta \sim+90^{\circ}$ $\left(n_{0, \theta} \sim 0.05\right.$ and $n_{1, \theta} \sim 0.06 n_{\mathrm{fan}, V}$. It is the features at about $\theta \sim-60^{\circ}$ and $\sim-170^{\circ}$ that most markedly disagree with the data from 2007-9. This will be discussed further in Section 4.2 .

Finally, in order to check whether the variation in density is due to the stellar background, the ring densities were plotted as a function of the ecliptic (not helioecliptic) longitude of the lines of sight to the centres of the images at mid-observation. If the ring densities are affected by features that are fixed with respect to the celestial sphere, they should be evident as consistent densities at given ecliptic longitudes between observations made at different times. Figure 14 shows very little evidence for such behaviour, with a spread in fitted densities across the range of ecliptic longitude. We conclude therefore that the main source of variation is not due to background sources.

\section{Discussion}

\subsection{Observations and data reduction}

The data presented here are high-sensitivity photometric observations of the Venus dust

ring taken using two different spacecraft from 2007-2014. Although study of the IPD cloud was not a primary scientific purpose of the HI-2 cameras, the data are useful for detailed studies of degree-scale features with low-surface brightness. However, the proportion of 

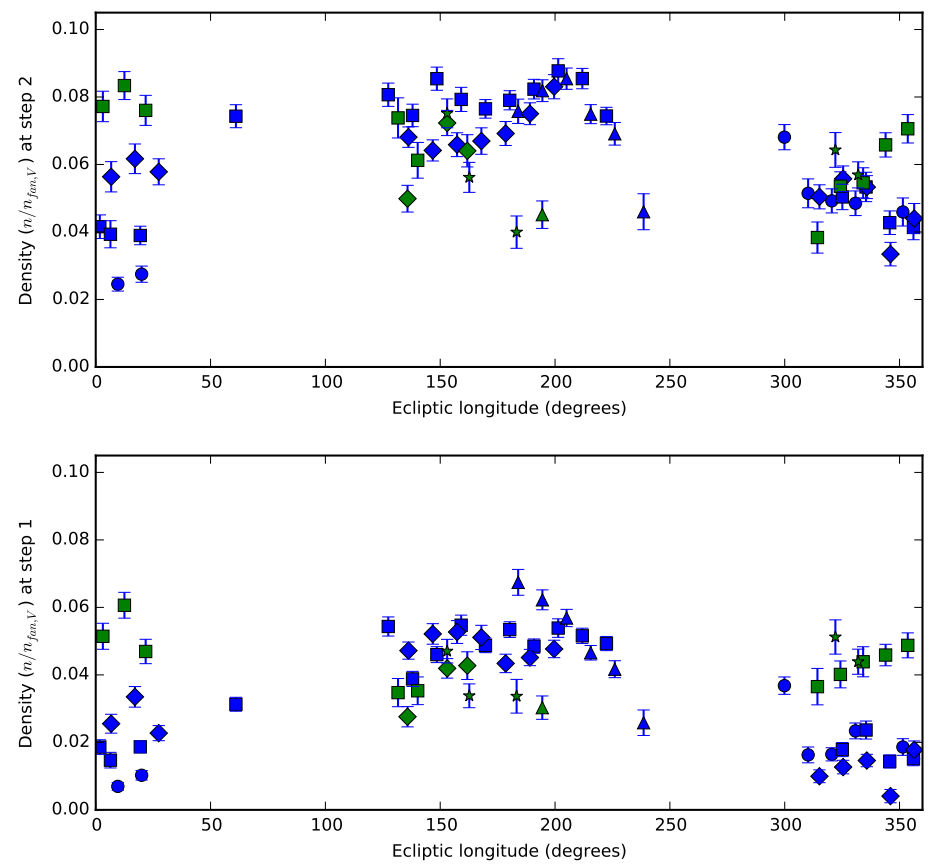

Fig. 14. - Fitted values of $n_{0, \theta}$ (lower panel) and $n_{1, \theta}$ (upper panel) plotted against ecliptic longitude $\lambda$ of the lines-of-sight for each image. HI-2A data are in blue, HI-2B data are in green. Key: o-2007, $\square-2008, \triangle-2009, \diamond-2013, \star-2014$. 
collected data that is suitable for the image processing techniques used here is rather low. From 8 years of observations using two spacecraft, we obtained 65 usable 10-day data sets, which corresponds to about 11 percent of the data. The major problem in using much of the data is the presence of bright sources within, or close to, the coarse grid area. This was particularly the case for the interval 2010-12, during which time the presence of the Earth in the fields-of-view of both HI-2A and HI-2B led to the rejection of all data from this time. Another problem is the sensitivity of the photometry to the presence of CMEs in the field of view. The period 2007-9 corresponded to solar minimum, and very few data sets from this time were rejected on the basis of CME activity or high residual noise levels. For the data from 2013-14, many more sets showed high noise levels. This is particularly unfortunate as it has resulted in a very sparse coverage of density variations in the ring at this later time.

With regard to the processing technique itself, we note that the method is not critically dependent on the value of the probability cut $P_{z}$. Any value of $P_{z}$ that acts as an estimator of surface brightness of the zodiacal light to within a constant offest value can potentially be used for this analysis. We note that JBB adopted a value of $P_{z}=0.45$, which although not optimal in terms of reducing the noise associated with the stellar background, yielded results that are very similar to those presented in this re-analysis.

\subsection{Properties of the Venus ring}

As noted above, the motivation for searching for a dust ring at Venus was the expectation that such a ring would arise from mean motion resonances between the planet and dust on exterior orbits. The radial location of the ring features close to the semi-major axis of Venus suggests that gravitational perturbation by Venus plays a key role in the formation of the ring. The inclination of the ring does however seem to be in a different plane to the orbit of Venus. This is likely to reflect the probability of capture of dust into 
resonant orbits, and this in turn, will depend on orientation of the symmetry plane of the IPD cloud in the vicinity of Venus, and the eccentricity of dust orbits in this location. With regard to the former, we note that near the Earth the IPD cloud has an inclination lower than that of the orbit of Venus, e.g. $i=2.03^{\circ}$ (Kelsall et al. 1998) and $i=1.35^{\circ}$ (Jones \& Rowan-Robinson 1993). With regard to the latter point, Tsumura et al. (2010) argue, on the basis of near-IR spectroscopy of IPD conducted with CIBER, that there is a significant component of asteroidal dust close to the symmetry plane of the zodiacal cloud. Such dust is likely to be on highly circularised orbits which would then be preferentially trapped into resonances.

To date, modelling of the interaction between dust in the zodiacal cloud and particular planets has concentrated on the Earth (Dermott et al. 1994; Mustill \& Wyatt 2011) and Mars (Mustill \& Wyatt 2011), but not on Venus. There are, however, some general observations that can be made, as well as reference to models of planet-dust interactions that have been made in the context of exozodiacal clouds. JBB noted that the amplitude of the density enhancements in the Venus ring, which they reported to be up to $\sim 0.1 n_{\mathrm{fan}, V}$, were less than the corresponding maximum enhancement of the Earth ring $\sim 0.15 n_{\text {fan, } E}$ (where the subscript $E$ denotes a dust cloud density at the Earth's orbit) (Kelsall et al. 1998). This is in qualitative agreement with the expectation that the higher Keplerian speeds near the orbit of Venus would lead to a lower probability of capture into resonant

orbits. The results of this more comprehensive study find a slightly lower maximum value of density enhancement at Venus $\sim 0.085 n_{\mathrm{fan}, V}$, but this remains consistent with JBB.

Simulations of planet-dust interactions (e.g. Stark \& Kuchner 2008) suggest that the azimuthal variation in density in a resonance ring might be expected to be fixed in a frame that is co-rotating with the planet. The variation of $n_{0, \theta}$ and $n_{1, \theta}$ against co-rotating longitude $\theta$ presented in Figures 12 and 13 suggest that this may not be the case for the 
Venus ring. In particular, it was noted in Section 3.3 that the clearest discrepancies in the pattern between the 2007-9 and 2013-4 data are features at $\theta \sim-70^{\circ}$ and $\theta \sim-170^{\circ}$.

Unfortunately, the coverage of $\theta$ in the 2013-4 data set is insufficient to allow a detailed matching between features observed at these different epochs, but it is possible to investigate whether a small change to the pattern speed can produce a better match between the data at these two epochs. This was done by plotting the parameters $n_{0, \theta}$ and $n_{1, \theta}$ against co-rotating longitude for a pattern speed corresponding to the mean motion $\left(n_{a}\right)$ for orbits with semi-major axes $(a)$ ranging from 0.69 to $0.75 \mathrm{AU}$.
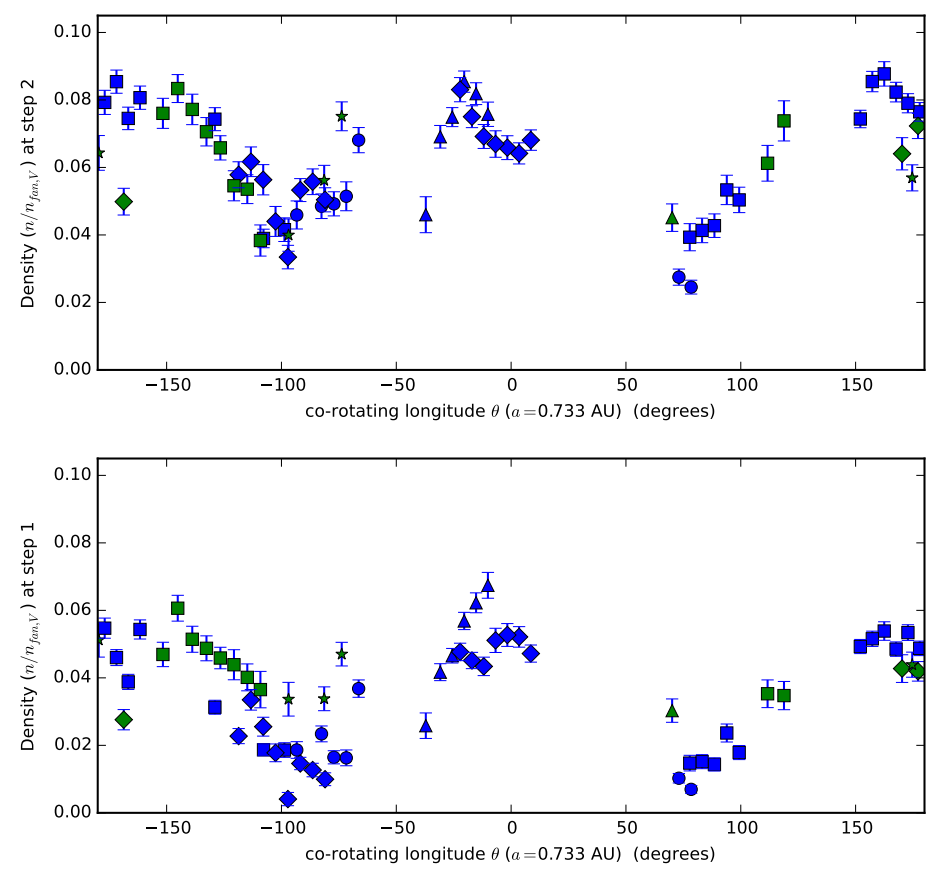

Fig. 15.- An example of the variation of azimuthal density parameters, $n_{0, \theta}$ (lower panel) and $n_{1, \theta}$ (upper panel) of the ring plotted against the co-rotating longitude with a pattern speed corresponding to the mean motion at $a=0.733 \mathrm{AU}$.

An example of a case where a qualitatively better fit to a smoothly varying density distribution is shown in Figure 15 for the case of $a=0.733 \mathrm{AU}\left(n_{a}=10.012 \mathrm{rad} \mathrm{yr}^{-1}\right)$. This represents the smallest difference in pattern speed that removes the discrepancies 
between the 2007-9 and the 2013-4 data sets.

A similar result (not shown) is obtained for the pattern speed corresponding to $a=0.711 \mathrm{AU}\left(n_{a}=10.480 \mathrm{rad} \mathrm{yr}^{-1}\right)$. Given that the 2013-4 data are so sparse, the significance of these possible pattern speeds should not be overstated, suffice to note that they do offer a possible explanation of discrepancies in the density profiles between the two epochs. It is also of interest that the semi-major axes of orbits corresponding to these pattern speeds are close to the radii of the inner and outer steps of the ring model adopted here. However, further observational data are needed to determine the pattern speed of the azimuthal density profile.

Notwithstanding the possible existence of a small difference between the ring pattern speed and the mean motion of Venus, the modulation of the density parameters around the ring is likely to provide information about the order of the dominant resonances that give rise to the ring. The 2007-9 data (Figure 12 provide reasonably good coverage of the whole ring, and seem to show that there are two minima in $n_{0, \theta}$ and $n_{1, \theta}$ (at $\theta \sim-100^{\circ}$ and $\sim+70^{\circ}$ ) that separate the two rather broad maxima. In the Case I resonance of Kuchner \& Holman (2003), such a pattern of two broad maxima separated by $180^{\circ}$ in co-rotating longitude would be indicative of a major contribution from dust in a $3: 2$ orbital resonance. However, the locations of the maxima from such a resonance would be expected to be close to $\theta= \pm 90^{\circ}$, which is a phase shift of about $90^{\circ}$ from what is observed. Of course, if the ring rotates with a pattern speed that differs from the orbital speed of Venus, as suggested above, there would be no expectation of finding density enchancements at any particular co-rotating longitude. It is the case, however, that the Case I resonance of Kuchner \& Holman (2003) should have a pattern that is fixed with respect to the mean motion of Venus.

With regard to the order of possible resonances giving rise to the Venus ring, Pástor 
(2016) has suggested that the narrow width of the Venus ring may be indicative of dust in a $1: 1$ resonance. We note that while the analysis used here, and by JBB, does pick out two characteristic radial distances $\left(r_{0}\right.$, and $\left.r_{1}\right)$, these are locations in which the ring density changes abruptly (in the radial direction). Indeed, since the ring density increases at both of these locations, the ring must be wider than the distance defined by $\left(r_{1}-r_{0}\right)$, and that our analysis is unable to discern where the outer edge of the ring lies.

\section{Conclusions}

In this paper we have identified all usable data sets acquired from the HI-2 instrument on both STEREO-A and -B in the interval from mission launch to the spacecraft passing behind the Sun. These data have been analysed to extract images that provide information about the Venus dust ring based on spatial variation of the column density of dust near the line of sight to the tangent of the ring. We have presented results from data acquired at two epochs: 2007-9 and 2013-14. There is more usable data from the former, a fact we attribute to the low incidence of CMEs close to solar minimum.

Analysis of images suggest that the orientation of the ring differs from that of the orbit of Venus. Specifically, the ring has a lower inclination: this might reflect the orientation of the IPD cloud close to Venus, or a higher capture probability for asteroidal grains near the ecliptic plane, or a combination of these factors. We have also investigated the azimuthal structure of the ring, and find that the pattern of dust distrubution in 2007-9 data supports a view that dust in a $3: 2$ orbital resonance makes a major contribution to the ring.

There are aspects of the behaviour of the ring density distribution that are not in accord with theoretical models. Specifically, the location of the density maxima around the ring are offset by about $90^{\circ}$ in co-rotating longitude from their expected positions (Kuchner 
\& Holman 2003). Also, comparison of 2007-9 data to 2013-14 data reveals changes in the density distribution with co-rotating longitude that might be indicative of a pattern speed for the ring that differs from the mean motion of Venus. Again, this would be unexpected, based on current models of resonance rings.

It is the case however that no detailed modelling of the interaction of Venus with the interplanetary dust cloud has yet been conducted. In particular, it would be useful to have simulations based on the physical and orbital characteristics of Venus that could be compared directly with observations of this sort.

We note that the STEREO spacecraft have now passed behind the Sun. Although STEREO-B is not currently functioning correctly, if STEREO-A remains operational, we would expect further useful data on the Venus dust ring to be acquired over the coming years. The data from solar minimum in around 2018-2020 should be particularly useful in providing good coverage of density changes with co-rotating longitude that can be compared with the data presented here. 


\section{REFERENCES}

Brown, D. S., Bewsher, D., \& Eyles, C. J., 2009, Solar Phys. 254, 185

Burns, A., Lamy, P. L., Soter, S., 1979, Icarus, 40, 1

Calabretta, M.R, \& Greisen, E. W. A\&A, 395, 1077

Dermott, S. F., Jayraraman, S., Xu, Y. L. , Gustafson, B. A. S., \& Liou, J. C. 1994, Nature, 369,719

Eyles, C. et al. 2009, Solar Phys., 254, 387

Grün, E., Zook, H. A., Fechtig, H., Giese, R. H., 1985, Icarus, 62, 244

Hahn, J. M., Zook, H. A. , Cooper, B. \& Sunkara, B., 2002, Icarus, 158, 360

Hong, S. S., 1985, A\&A, 146, 67

Jackson,A. A. \& Zook, H. A. 1989, Nature, 337, 629

Jones, M.H., Bewsher D., Brown D.S. 2013, Science, 342, 960.

Jones M.H., \& Rowan-Robinson, M., 1993, MNRAS, 264, 237.

Kaiser, M. L. et al., 2008, Space Sci. Rev., 136, 5

Kelsall, T. et al., 1998, ApJ, 508, 44

Koutchmy, S. , \& Lamy, P. L., in Properties and interactions of interplanetary dust, R. H. Giese, P. Lamy, Eds. (Reidel, Dordrecht, 1985). pp. 63-74.

Kuchner, M. J., Reach, W. T. \& Brown, M. E., 2000, Icarus, 145, 44

Kuchner, M. J. \& Holman, M. J., 2003, ApJ, 588, 1110 
Leinert, C. \& Moster, B., 2007, A\&A, 472, 335

Lynds, B.T., 1965, ApJS, 12, 163L

Mustill, A. J., \& Wyatt, M. C., 2011, MNRAS, 413, 554

Nesvorný, D. et al., 2010, ApJ, 713, 816

Pástor, P., 2016, MNRAS, 460, 524

Reach, W. T. et al., 1995, Nature, 374, 521

Reach, W. T., 2010, Icarus, 209, 848

Rowan-Robinson, M., \& May, B., 2013, MNRAS, 429, 2894

Stark, C. C., \& Kuchner, M. J. 2008, ApJ, 686, 637

Tappin, S.J., Eyles, C.J., \& Davies, J.A. 2015, Solar Phys., 290, 2143

Thompson, W.T., 2006, A\&A, 449, 791

Tsumura, K., Battle, J., Bock, J., et al. 2010, ApJ, 719, 394

Weidenschilling, S. J., \& Jackson A. A., 1993, Icarus, 104, 244 


\begin{tabular}{|c|c|c|c|c|c|}
\hline $\begin{array}{l}\text { Name of } \\
\text { data set }\end{array}$ & Start & $\begin{array}{l}\text { Mid-obs } \\
\text { (JD) }\end{array}$ & $\begin{array}{c}\lambda_{T} \\
\text { (degrees) }\end{array}$ & $\begin{array}{c}\theta_{V} \\
\text { (degrees) }\end{array}$ & Notes \\
\hline A070106 & 2007-Jan-06 00:00:00 & 2454111.5 & 68.5 & 95.0 & Rejected (high noise level) \\
\hline A070205 & 2007-Feb-05 00:00:00 & 2454141.5 & 99.6 & 78.4 & \\
\hline A070215 & 2007-Feb-15 00:00:00 & 2454151.5 & 110.0 & 72.8 & \\
\hline A070308 & 2007-Mar-08 00:00:00 & 2454172.5 & 131.8 & 60.9 & Rejected (LBN in field) \\
\hline A071109 & 2007-Nov-09 00:00:00 & 2454418.5 & 29.9 & -75.4 & \\
\hline A071119 & 2007-Nov-19 00:00:00 & 2454428.5 & 40.2 & -81.3 & \\
\hline $\mathrm{A} 071129^{\dagger}$ & 2007-Nov-29 00:00:00 & 2454438.5 & 50.5 & -87.3 & \\
\hline A071209 & 2007-Dec-09 00:00:00 & 2454448.5 & 60.8 & -93.2 & \\
\hline A071229 & 2007-Dec-29 00:00:00 & 2454468.5 & 81.5 & -105.0 & \\
\hline A080108 & 2008-Jan-08 00:00:00 & 2454478.5 & 91.8 & -110.8 & \\
\hline A080125 & 2008-Jan-25 00:00:00 & 2454495.5 & 109.4 & -120.5 & \\
\hline A080204 & 2008-Feb-04 00:00:00 & 2454505.5 & 119.8 & -126.0 & Rejected (LBN in field) \\
\hline A080214 ${ }^{\dagger}$ & 2008-Feb-14 00:00:00 & 2454515.5 & 130.2 & -131.5 & Rejected (LBN in field) \\
\hline A080224 & 2008-Feb-24 00:00:00 & 2454525.5 & 140.6 & -136.9 & Rejected (LBN in field) \\
\hline A080305 & 2008-Mar-05 00:00:00 & 2454535.5 & 151.0 & -142.3 & \\
\hline A080507 & 2008-Мay-07 00:00:00 & 2454598.5 & 217.4 & -176.0 & \\
\hline A080517 & 2008-May-17 00:00:00 & 2454608.5 & 228.0 & 178.6 & \\
\hline A080527 & 2008-May-27 00:00:00 & 2454618.5 & 238.5 & 173.1 & \\
\hline $\mathrm{A} 080606^{\dagger}$ & 2008-Jun-06 00:00:00 & 2454628.5 & 249.1 & 167.6 & \\
\hline A080616 & 2008-Jun-16 00:00:00 & 2454638.5 & 259.7 & 162.0 & \\
\hline A080626 & 2008-Jun-26 00:00:00 & 2454648.5 & 270.3 & 156.4 & \\
\hline A080706 & 2008-Jul-06 00:00:00 & 2454658.5 & 280.8 & 150.7 & \\
\hline A080716 & 2008-Jul-16 00:00:00 & 2454668.5 & 291.4 & 145.0 & \\
\hline A080726 & 2008-Jul-26 00:00:00 & 2454678.5 & 301.9 & 139.2 & \\
\hline A080805 ${ }^{\dagger}$ & 2008-Aug-05 00:00:00 & 2454688.5 & 312.4 & 133.5 & \\
\hline A081112 & 2008-Nov-12 00:00:00 & 2454787.5 & 55.1 & 78.6 & \\
\hline A081122 & 2008-Nov-22 00:00:00 & 2454797.5 & 65.4 & 73.0 & \\
\hline A081202 & 2008-Dec-02 00:00:00 & 2454807.5 & 75.7 & 67.4 & \\
\hline A081212 & 2008-Dec-12 00:00:00 & 2454817.5 & 86.1 & 61.8 & \\
\hline A081222 & 2008-Dec-22 00:00:00 & 2454827.5 & 96.4 & 56.2 & \\
\hline A090609 ${ }^{\dagger}$ & 2009-Jun-09 00:00:00 & 2454996.5 & 273.9 & -37.7 & \\
\hline A090619 & 2009-Jun-19 00:00:00 & 2455006.5 & 284.5 & -43.0 & \\
\hline A090629 & 2009-Jun-29 00:00:00 & 2455016.5 & 295.0 & -48.3 & \\
\hline A090709 & 2009-Jul-09 00:00:00 & 2455026.5 & 305.5 & -53.7 & \\
\hline A090719 & 2009-Jul-19 00:00:00 & 2455036.5 & 316.0 & -59.1 & \\
\hline A090731 & 2009-Jul-31 00:00:00 & 2455048.5 & 328.6 & -65.8 & \\
\hline A120404 ${ }^{\dagger}$ & 2012-Apr-04 00:00:00 & 2456026.5 & 270.1 & 107.8 & Rejected (ghost image) \\
\hline A130201 & 2013-Feb-01 00:00:00 & 2456329.5 & 226.2 & -61.5 & \\
\hline A130211 & 2013-Feb-11 00:00:00 & 2456339.5 & 236.8 & -66.7 & \\
\hline A130221 & 2013-Feb-21 00:00:00 & 2456349.5 & 247.3 & -71.9 & \\
\hline A130303 & 2013-Mar-03 00:00:00 & 2456359.5 & 257.9 & -77.2 & \\
\hline A130313 & 2013-Mar-13 00:00:00 & 2456369.5 & 268.5 & -82.5 & \\
\hline $\mathrm{A} 130323^{\dagger}$ & 2013-Mar-23 00:00:00 & 2456379.5 & 279.1 & -87.8 & \\
\hline A130402 & 2013-Apr-02 00:00:00 & 2456389.5 & 289.6 & -93.2 & \\
\hline A130722 & 2013-Jul-22 00:00:00 & 2456500.5 & 45.1 & -157.0 & \\
\hline A130801 & 2013-Aug-01 00:00:00 & 2456510.5 & 55.4 & -162.7 & \\
\hline A130811 & 2013-Aug-11 00:00:00 & 2456520.5 & 65.7 & -168.4 & \\
\hline A130821 & 2013-Aug-21 00:00:00 & 2456530.5 & 76.1 & -174.0 & \\
\hline $\mathrm{A} 130831^{\dagger}$ & 2013-Aug-31 00:00:00 & 2456540.5 & 86.4 & -179.6 & \\
\hline A130910 & 2013-Sep-10 00:00:00 & 2456550.5 & 96.7 & 174.9 & \\
\hline A130920 & 2013-Sep-20 00:00:00 & 2456560.5 & 107.1 & 169.5 & \\
\hline A130930 & 2013-Sep-30 00:00:00 & 2456570.5 & 117.4 & 164.0 & \\
\hline
\end{tabular}

Table 1: A summary of the data sets from STEREO-A selected for the creation of high sensitivity maps. LBN refers to bright nebulae from the catalogue of Lynds (1965). Data sets marked by $\dagger$ are those used to determine the scaling of the reference fan model (see text for details). 


\begin{tabular}{|c|c|c|c|c|c|}
\hline $\begin{array}{l}\text { Name of } \\
\text { data set }\end{array}$ & Start & $\begin{array}{c}\text { Mid-obs } \\
(\mathrm{JD})\end{array}$ & $\begin{array}{c}\lambda_{T} \\
\text { (degrees) } \\
\end{array}$ & $\begin{array}{c}\theta_{V} \\
\text { (degrees) }\end{array}$ & Notes \\
\hline B080408 & 2008-Apr-08 00:00:00 & 2454569.5 & 224.3 & -122.9 & \\
\hline B080418 & 2008-Apr-18 00:00:00 & 2454579.5 & 234.3 & -128.8 & \\
\hline B080428 & 2008-Apr-28 00:00:00 & 2454589.5 & 244.2 & -134.8 & \\
\hline B080508 & 2008-May-08 00:00:00 & 2454599.5 & 253.9 & -141.0 & \\
\hline B080518 & 2008-May-18 00:00:00 & 2454609.5 & 263.6 & -147.4 & \\
\hline B080528 & 2008-May-28 00:00:00 & 2454619.5 & 273.1 & -153.9 & \\
\hline B080607 & 2008-Jun-07 00:00:00 & 2454629.5 & 282.5 & -160.7 & \\
\hline B080617 & 2008-Jun-17 00:00:00 & 2454639.5 & 291.7 & -167.6 & \\
\hline B080627 & 2008-Jun-27 00:00:00 & 2454649.5 & 300.9 & -174.7 & Rejected (LBN in field) \\
\hline B080707 & 2008-Jul-07 00:00:00 & 2454659.5 & 309.9 & 178.1 & Rejected (LBN in field) \\
\hline B080717 & 2008-Jul-17 00:00:00 & 2454669.5 & 318.7 & 170.7 & Rejected (LBN in field) \\
\hline B081022 & 2008-Oct-22 00:00:00 & 2454766.5 & 41.6 & 98.3 & \\
\hline B081101 & 2008-Nov-01 00:00:00 & 2454776.5 & 50.2 & 91.1 & \\
\hline B081111 & 2008-Nov-11 00:00:00 & 2454786.5 & 58.8 & 83.9 & Rejected (Poor detrending) \\
\hline B081121 & 2008-Nov-21 00:00:00 & 2454796.5 & 67.5 & 76.7 & Rejected (Poor detrending) \\
\hline B081201 & 2008-Dec-01 00:00:00 & 2454806.5 & 76.4 & 69.6 & Rejected (Poor detrending) \\
\hline B081211 & 2008-Dec-11 00:00:00 & 2454816.5 & 85.3 & 62.6 & Rejected (Poor detrending) \\
\hline B081221 & 2008-Dec-21 00:00:00 & 2454826.5 & 94.3 & 55.7 & Rejected (Poor detrending) \\
\hline B090101 & 2009-Jan-01 00:00:00 & 2454837.5 & 104.4 & 48.2 & \\
\hline B130130 & 2013-Jan-30 00:00:00 & 2456327.5 & 45.8 & 121.3 & \\
\hline B130209 & 2013-Feb-09 00:00:00 & 2456337.5 & 54.4 & 114.1 & Rejected (high noise level) \\
\hline B130219 & 2013-Feb-19 00:00:00 & 2456347.5 & 63.0 & 106.9 & \\
\hline B130301 & 2013-Mar-01 00:00:00 & 2456357.5 & 71.8 & 99.8 & \\
\hline B130325 & 2013-Mar-25 00:00:00 & 2456381.5 & 93.3 & 83.2 & Rejected (high noise level) \\
\hline B130404 & 2013-Apr-04 00:00:00 & 2456391.5 & 102.4 & 76.4 & Rejected (high noise level) \\
\hline B130424 & 2013-Apr-24 00:00:00 & 2456411.5 & 121.2 & 63.2 & Rejected (high noise level) \\
\hline B140315 & 2014-Mar-15 00:00:00 & 2456736.5 & 63.0 & -157.2 & \\
\hline B140326 & 2014-Mar-26 00:00:00 & 2456747.5 & 72.7 & -165.2 & \\
\hline B140408 & 2014-Apr-08 00:00:00 & 2456760.5 & 84.2 & -174.3 & Rejected (high noise level) \\
\hline B140418 & 2014-Apr-18 00:00:00 & 2456770.5 & 93.3 & 178.9 & \\
\hline B140606 & 2014-Jun-06 00:00:00 & 2456819.5 & 139.5 & 147.6 & Rejected (high noise level) \\
\hline B140827 & 2014-Aug-27 00:00:00 & 2456901.5 & 222.0 & 98.4 & Rejected (high noise level) \\
\hline B140906 & 2014-Sep-06 00:00:00 & 2456911.5 & 232.1 & 92.2 & \\
\hline B140916 & 2014-Sep-16 00:00:00 & 2456921.5 & 242.0 & 85.8 & \\
\hline
\end{tabular}

Table 2: A summary of the data sets from STEREO-B selected for the creation of high sensitivity maps. LBN refers to bright nebulae from the catalogue of Lynds (1965). 